\title{
Rational Engineering of Hydratase from Lactobacillus Acidophilus Reveals Critical Residues Directing Substrate Specificity and Regioselectivity
}

Eser, Bekir Engin; Poborsky, Michal; Dai, Rongrong; Kishino, Shigenobu; Ljubic, Anita; Takeuchi, Michiki; Jacobsen, Charlotte; Ogawa, Jun; Kristensen, Peter; Guo, Zheng

\section{Published in:}

ChemBioChem

Link to article, DOI:

10.1002/cbic.201900389

Publication date:

2020

Document Version

Peer reviewed version

Link back to DTU Orbit

Citation (APA):

Eser, B. E., Poborsky, M., Dai, R., Kishino, S., Ljubic, A., Takeuchi, M., Jacobsen, C., Ogawa, J., Kristensen, P., \& Guo, Z. (2020). Rational Engineering of Hydratase from Lactobacillus Acidophilus Reveals Critical Residues Directing Substrate Specificity and Regioselectivity. ChemBioChem, 21(4), 550-563. https://doi.org/10.1002/cbic.201900389

\section{General rights}

Copyright and moral rights for the publications made accessible in the public portal are retained by the authors and/or other copyright owners and it is a condition of accessing publications that users recognise and abide by the legal requirements associated with these rights.

- Users may download and print one copy of any publication from the public portal for the purpose of private study or research.

- You may not further distribute the material or use it for any profit-making activity or commercial gain

- You may freely distribute the URL identifying the publication in the public portal 


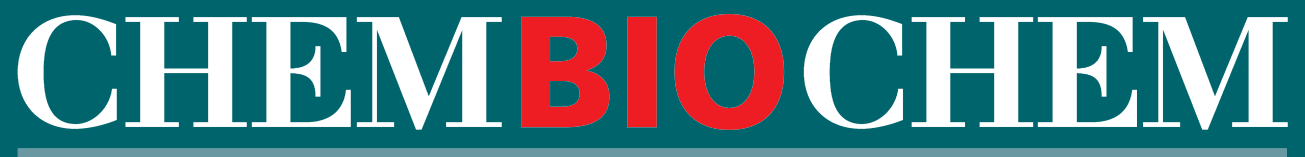

SYNTHETIC BIOLOGY \& BIO-NANOTECHNOLOGY

\section{Accepted Article}

Title: Rational Engineering of Hydratase from Lactobacillus Acidophilus Reveals Critical Residues Directing Substrate Specificity and Regioselectivity

Authors: Bekir Engin Eser, Michal Poborsky, Rongrong Dai, Shigenobu Kishino, Anita Ljubic, Michiki Takeuchi, Charlotte Jacobsen, Jun Ogawa, Peter Kristensen, and Zheng Guo

This manuscript has been accepted after peer review and appears as an Accepted Article online prior to editing, proofing, and formal publication of the final Version of Record (VoR). This work is currently citable by using the Digital Object Identifier (DOI) given below. The VoR will be published online in Early View as soon as possible and may be different to this Accepted Article as a result of editing. Readers should obtain the VoR from the journal website shown below when it is published to ensure accuracy of information. The authors are responsible for the content of this Accepted Article.

To be cited as: ChemBioChem 10.1002/cbic.201900389

Link to VoR: http://dx.doi.org/10.1002/cbic.201900389 


\section{Rational Engineering of Hydratase from Lactobacillus}

\section{Acidophilus Reveals Critical Residues Directing Substrate}

\section{Specificity and Regioselectivity}

Dr. Bekir Engin Eser ${ }^{1}$, Michal Poborsky ${ }^{1}$, Rongrong Dai ${ }^{1}$, Dr. Shigenobu Kishino ${ }^{2}$, Anita Ljubic ${ }^{3}$, Dr. Michiki Takeuchi ${ }^{2}$, Prof. Dr. Charlotte Jacobsen ${ }^{3}$, Prof. Dr. Jun Ogawa², Prof. Dr. Peter Kristensen ${ }^{4}$, Prof. Dr. Zheng Guo ${ }^{1^{*}}$

${ }^{1}$ Department of Engineering, Aarhus University, 8000 Aarhus, Denmark, ${ }^{2}$ Division of Applied Life Sciences, Graduate School of Agriculture, Kyoto University, Kyoto 606-8502, Japan, ${ }^{3}$ Division of Food Technology, National Food Institute, Technical University of Denmark, 2800 Kgs. Lyngby, Denmark, ${ }^{4}$ Faculty of Engineering and Science, Department of Chemistry and Bioscience, Aalborg University, 9220 Aalborg, Denmark

*Corresponding Author: Zheng Guo (e-mail: guo@eng.au.dk) 


\begin{abstract}
Enzymatic conversion of fatty acids (FAs) by fatty acid hydratases (FAHs) presents a green and efficient route for high-value hydroxy fatty acid (HFA) production. However, limited diversity was achieved among HFAs to date with respect to chain length and hydroxy position. In this study, two highly similar FAHs from Lactobacillus acidophilus were compared: FA-HY2 has narrow substrate scope and strict regioselectivity, whereas FA-HY1 utilizes longer chain substrates and hydrate various double bond positions. We reveal three active-site residues that play remarkable role in directing substrate specificity and regioselectivity of hydration. When these residues on FAHY2 are mutated to the corresponding ones in FA-HY1, we observed a significant expansion of substrate scope and a distinct enhancement in hydration of double bonds towards $\omega$-end of FAs. A three-residue mutant of FA-HY2 (TM-FA-HY2) displayed an impressive reversal of regioselectivity towards linoleic acid, shifting ratio of the HFA regioisomers (10-OH:13-OH) from 99:1 to $12: 88$. Notable changes in regioselectivity were also observed for arachidonic acid and for C18 PUFA substrates. In addition, TM-FA-HY2 converted EPA to its 12-hydroxy product with high conversion at preparative scale. Furthermore, we demonstrated microalgae as a source of diverse FAs for HFA production. Our study paves the way for tailor-made FAH design enabling production of diverse HFAs for various applications from polymer industry to medical field.
\end{abstract}




\section{INTRODUCTION}

Hydroxy fatty acids (HFAs) are unique fatty acid (FA) derivatives with beneficial medical properties $^{[1]}$ as well as with various industrial uses ${ }^{[2]}$. HFAs can occur as gut microbial FA metabolites and suggested to be involved in the regulation of host energy metabolism ${ }^{[1 \mathrm{a}]}$. Such a metabolite, 10-hydroxy-cis-12-octadecenoic acid, exhibited anti-inflammatory effects in murine enterocytes in vitro ${ }^{[1 \mathrm{~b}]}$ and suppressed intestinal inflammation in animal models ${ }^{[3]}$. Recently described branched FA esters of HFAs (FAHFAs) are naturally occurring bioactive lipids with anti-diabetic and anti-inflammatory effects ${ }^{[4]}$. More than 20 families of FAHFAs were identified in human body that differ by composition of their FAs and position of the ester bond between HFA and $\mathrm{FA}^{[4 \mathrm{~b}]}$. Specialized proresolving mediators (SPM) are another group of HFA metabolites that exhibit effects such as resolution of inflammation, stimulation of tissue regeneration and tumor growth suppression ${ }^{[5]}$. SPMs include lipoxins, which are tri-hydroxy derivatives of arachidonic acid (AA) and resolvins, which are di-, tri-, or poly-hydroxy derivatives of mainly eicosapentaenoic acid (EPA) and docosahexaenoic acid (DHA ${ }^{[5 \mathrm{e}]}$. Moreover, it was demonstrated that lactobacilli used for food fermentation produce anti-fungal HFAs ${ }^{[6]}$. The potency of these HFAs against fungi was dependent on their structure, including the position and configuration of the hydroxy group ${ }^{[6 b]}$. Ricinoleic acid (12-hydroxy-9-cis-octadecenoic acid) is used in several industries for manufacturing of various products such as surfactants, plastics (Nylon-11), plasticizers, polyurethane resins, emulsifiers and lubricant additives ${ }^{[2 \mathrm{c}, 7]}$. 12-hydroxystearic acid is commonly used in skin care preparations ${ }^{[8]}$. HFAs can also be used to produce polymers for durable and flexible bioplastics with properties that can be tailored for a specific application ${ }^{[9]}$. The FA length and the position of the hydroxyl dictate the size of the monomers and as such, the overall properties of the material. 
Castor plants are used to produce the most common industrial HFA, ricinoleic acid, but they do not provide any further product diversity and accumulate toxic $\operatorname{ricin}^{[2 \mathrm{a}, 2 \mathrm{c}, 10]}$. Due to poor reactivity of water, chemical methods for hydration of isolated carbon-carbon double bonds often require harsh reaction conditions such as transition metal and/or acid-base catalysts, high temperatures and hazardous organic solvents ${ }^{[11]}$. Besides, chemical methods lead to a variety of undesired side reactions and do not offer the necessary stereo- and regioselectivity to unlock the full potential of $\mathrm{HFAs}^{[2 \mathrm{~b}, 12]}$. There are a few chemical methods presented in literature for stereoselective addition of water to unsaturated ketones ${ }^{[13]}$ and $\mathrm{FAs}^{[14]}$, however, carbon-carbon double bond needs to be activated as an $\alpha, \beta$-unsaturated carbonyl and transition metal catalysts are required. Fatty acid hydratases (FAHs) present an alternative enzymatic route for synthesis of HFAs. These enzymes attracted great interest in recent years due to their high biocatalytic potential ${ }^{[2 a, 2 b, 12,15]}$. FAHs can act on isolated carbon-carbon double bonds of the FA and produce HFAs by reversible addition of water onto a double bond of an unsaturated FA in a regio- and stereospecific manner ${ }^{[12,15 b]}$ (Scheme 1). Although other enzymatic routes are present for hydration of activated $\alpha, \beta$-unsaturated carbonyl compounds, FAHs can efficiently act on non-activated carbon-carbon double bonds ${ }^{[2 b]}$. Moreover, regio- and stereoselective hydration of non-FA substrates, including terminal and internal alkenes as well as FA derivatives, have been recently demonstrated by engineered $\mathrm{FAHs}^{[11 \mathrm{c}, 16]}$. Although no redox chemistry is involved, FAD is essential for this reaction and most likely plays a structural or charge-stabilization role ${ }^{[17]}$. Advantageously for a biocatalytic process, FAD is required only in catalytic amount and no recycling of the cofactor is needed. Moreover, hydratases are highly active and relatively stable enzymes, in comparison to other hydroxylases such as $\mathrm{P} 450$ monooxygenases and lipoxygenases, and can be expressed in high quantities in $E$. coli host ${ }^{[12]}$. Utilizing unsaturated 
FAs as substrates, hydratases are also well suited to convert algae or plant oil hydrolysates as well as waste oil into valuable chemicals.

Scheme 1. Reaction catalyzed by Fatty Acid Hydratases

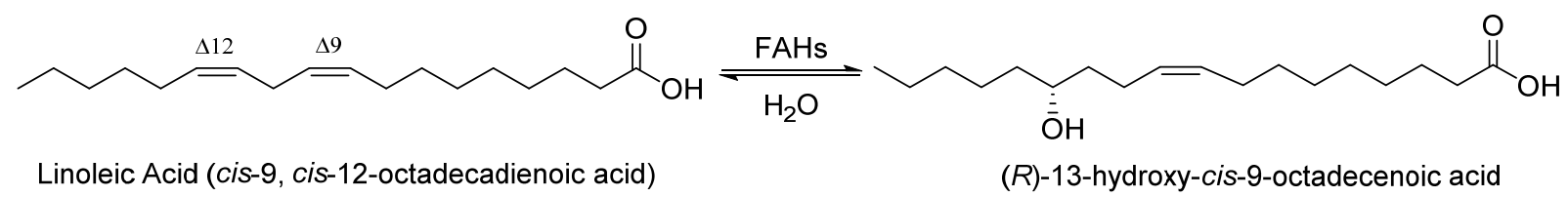

Most hydratases are active towards C16-C18 unsaturated FAs and are only able to add water on $\Delta 9$ double bonds, with oleic acid (OA) being the most preferred substrate. Broadening the substrate range and regiodiversity of hydratases will improve their applicability for industrial and medical fields. Recently, two novel hydratases (FA-HY1 and FA-HY2) from L. acidophilus, with 76\% sequence similarity, were described ${ }^{[18]}$. Where FA-HY2 is a typical hydratase, FA-HY1 is rather unique, with a broad substrate promiscuity, as it accepts substrates up to 22 carbons in length and preferably hydrates double bonds other than $\Delta 9^{[18 \mathrm{a}]}$.

In this study, active-sites of FA-HY1 and FA-HY2 were compared to identify residues that dictate the dramatic difference in substrate specificity and regioselectivity. Our analysis was based on the structure of a closely related homolog, OhyA (oleate hydratase from Elizabethkingia meningoseptica ${ }^{[17]}$ and sequence comparison between FA-HY1, FA-HY2 and OhyA. Through site-directed mutagenesis, we targeted three residues of FA-HY2, located around the carboxylate end of FA substrate, and substituted them into the corresponding amino acids from FA-HY1, either individually or in combination. Our results demonstrate that mutations, in a stepwise manner from a single residue mutant to a triple mutant (TM-FA-HY2), exhibit increasing degree of changes in substrate preference and in regioselectivity, converting FA-HY2 to a more versatile catalyst with similar properties to FA-HY1. Regioselectivity shifts were observed to some extent for all C18 
PUFAs and arachidonic acid (AA) with TM-FA-HY2 variant as well as a double mutant. Moreover, TM-FA-HY2 produced 12-OH products from cis-5, cis-8, cis-11, cis-14, cis-17-eicosapentaenoic acid (EPA) and AA more efficiently than the most active wild-type enzyme for the same products. These two 12-OH products were detected for the first time with a hydratase. The practical utility of FA-HY1, FA-HY2 and mutant variants for production of various HFAs through preparative scale reactions was also demonstrated. Furthermore, our steady-state kinetic data indicate that the effect of mutations is exhibited mostly on $k_{\mathrm{cat}}$ rather than $K_{\mathrm{m}}$. Substrate docking studies further highlight the significance of positioning of double bond for activity and regioselectivity. Lastly, production of HFAs by hydratases from FA of two microalgae species demonstrates microalgae as a sustainable source of FAs for production of value-added HFAs.

\section{MATERIALS AND METHODS}

\section{Chemicals}

All FAs were purchased from Sigma-Aldrich, except EPA (Calbiochem) and cis-Vaccenic acid (MP Biomedicals). All FA substrate and standards were of the purest grade available (at least 98.5\% purity). N, O-Bis(trimethylsilyl)trifluoroacetamide with trimethylchlorosilane (BSTFA + TMCS; 99:1) used for silylation and FAD used as cofactor were also purchased from SigmaAldrich. All other common chemicals were analytical grade and are commercially available.

\section{Site-directed Mutagenesis}

The genes fa-hy1(GenBank ID: LC030242.1) and fa-hy2 (genebank ID LC030243.1) from the strain Lactobacillus acidophilus NTV001 were 1,773 and 1,776 base pairs (bp) in length, respectively, and both genes (including stop codons) were cloned into pET-21b vector using XhoI and NdeI endonucleases as previously described ${ }^{[18 a]}$. All forward and reverse primers (see Table S8 for sequences) required for mutagenesis were obtained from Sigma-Aldrich. Site-directed 
mutagenesis was carried out using QuikChange II Site-Directed Mutagenesis Kit from Agilent. The success of the mutagenesis was confirmed by sequencing (Eurofins Genomics). For plasmid transformation and amplification, the chemically competent E. coli strain XL1-Blue (Agilent) was used. Plasmid purification was performed using GeneJET plasmid miniprep kit (Thermo Fisher Scientific).

\section{Protein Expression and Purification}

For protein expression, E.coli Rosetta (DE3) cells (Merck Millipore) were transformed with a plasmid possessing the proper gene. $0.5 \mathrm{~L}$ or $1 \mathrm{~L}$ of $2 \mathrm{xTY}$ or $\mathrm{LB}$ media (with $100 \mu \mathrm{g} / \mathrm{mL}$ ampicillin) was inoculated with 5-10 mL of starter culture and the cells were grown until the optical density at $600 \mathrm{~nm}$ reached $0.5-1.0$. At this point, the expression was induced by addition of $0.1 \mathrm{mM}$ isopropyl$\beta$-D-thiogalactopyranoside (IPTG). Afterwards, the expression was carried at $18{ }^{\circ} \mathrm{C}$ and $180 \mathrm{rpm}$ for 16-20 hours. The cells were then harvested by centrifugation at $4,000 \mathrm{~g}$ and $4{ }^{\circ} \mathrm{C}$ for $30 \mathrm{~min}$, washed twice with $0.85 \%$ solution of $\mathrm{NaCl}$. The cell pellet was stored at $-80{ }^{\circ} \mathrm{C}$ until further use.

All the steps below were carried at $4{ }^{\circ} \mathrm{C}$, unless otherwise stated. The previously washed cells were resuspended in $100 \mathrm{mM}$ potassium phosphate buffer, $\mathrm{pH} 6.0(5 \mathrm{~mL}$ per $0.5 \mathrm{~L}$ of culture $)$, and supplemented with cOmplete EDTA-Free Protease Inhibitor (Sigma-Aldrich). After cell disruption with sonication, the cell debris was pelleted by centrifugation at $30,000 \mathrm{~g}$ for 2 hours and the supernatant was stored at $-80^{\circ} \mathrm{C}$.

All proteins were purified at room temperature with ÄKTA explorer fast protein liquid chromatography (FPLC) system (GE Healthcare) equipped with anion-exchange Mono Q HR 5/5 column (GE Healthcare). The proteins were eluted using a linear gradient of $\mathrm{NaCl}$ from 0 to $1 \mathrm{M}$ in $50 \mathrm{mM}$ potassium phosphate buffer at $\mathrm{pH} 6.0$ with a flow-rate of $2 \mathrm{~mL} / \mathrm{min}$. Only FA-HY1 required two step purification, with a subsequent loading onto cation-exchange SP Sepharose HP 
column (GE Healthcare) and elution under the same condition as previously. The eluted fractions were analyzed by SDS-PAGE and those showing high purity of desired protein band were collected. The pooled fractions were further buffer exchanged (into $100 \mathrm{mM}$ potassium phosphate at $\mathrm{pH}$ 6.0) and concentrated using centrifugal filters with 10 or $30 \mathrm{kDa}$ cut-off. The concentration of purified enzymes were determined with BCA Protein Assay Kit (Pierce, Thermo Fisher Scientific). All enzyme solutions were aliquoted and stored at $-80^{\circ} \mathrm{C}$.

\section{Enzyme Activity Assays and Product Extraction}

For analysis of substrate specificity and regioselectivity, a standard enzyme reaction mixture contained (in a final volume of $1 \mathrm{~mL}), 0.5 \mathrm{mg}$ of a FA substrate (1.5-2.0 mM, depending on molecular weight of FA) and $300 \mu \mathrm{g}$ of enzyme $(4.5 \mu \mathrm{M})$ in $100 \mathrm{mM}$ potassium phosphate buffer, $\mathrm{pH}$ 6.0. The mixture also consisted of $5 \%$ ethanol to solubilize the FAs and $0.1 \mathrm{mM}$ FAD cofactor. The assays were incubated at $37^{\circ} \mathrm{C}$ with a shaking speed of $120 \mathrm{rpm}$ for about 16 hours. Incubation of the assays were performed in an airtight box, in which an anaerobic atmosphere was maintained by Oxoid AnaeroGen sachets (Thermo Fisher Scientific), to prevent oxidation of unsaturated FAs. Reactions were quenched by addition of $1 \mathrm{~mL}$ of chloroform followed by rigorous mixing. Kinetic assays were initiated by addition of enzyme and quenched by chloroform. For short time steadystate assays (less than $30 \mathrm{~min}$ ), reactions were carried out aerobically, but for longer time assays anaerobic conditions were maintained. Initial velocities obtained from each substrate concentration was fitted to Michaelis-Menten equation using Kaleidagraph Program (Synergy software). The enzyme concentration and assay time used for each steady-state analysis is given in Supporting Information (Table S1) along with Michaelis-Menten plots.

After the enzyme reaction is quenched, $1 \mathrm{mg}$ heptadecanoic or hexadecanoic acid internal standard was added to each reaction mixture and Bligh-Dyer method was then used to extract the 
lipid components. Chloroform, methanol and $1.5 \% \mathrm{KCl}$ solution (with a ratio of $2: 2: 1$, respectively) in a total volume of $5 \mathrm{~mL}$ was added onto $1 \mathrm{~mL}$ of the assay mixture. Each chemical was added separately and the mixture was vortexed at highest speed for 5-10 s after each addition. In order to separate the phases, the mixture was centrifuged at $3,000 \mathrm{~g}$ for $10 \mathrm{~min}$ at room temperature. The bottom chloroform phase was then collected in a fresh glass tube and subsequently, the solvent was completely evaporated by a gentle nitrogen flow.

\section{Preparative Scale Reactions and Product Purification}

All preparative scale reactions were set at a scale of $100 \mathrm{~mL}$ total reaction volume in Erlenmeyer flasks that were placed in an airtight box with AnaeroGen sachets. Initial time course experiments at smaller scale (about $4 \mathrm{~mL}$ ) were performed to determine optimum conditions and concentrations for preparative scale assays (Figure S47). Reaction mixtures were incubated at $37{ }^{\circ} \mathrm{C}$ for about 24 hours with gentle shaking at $100 \mathrm{rpm}$. A standard preparative scale reaction mixture contained (in a final volume of $100 \mathrm{~mL}), 50-100 \mathrm{mg}$ of a FA substrate (1.5-3.5 mM final concentration; 0.15$0.35 \mathrm{mmol}$ scale) and 10-60 $\mathrm{mg}$ of enzyme (1.5-9.0 $\mu \mathrm{M})$ in $100 \mathrm{mM}$ potassium phosphate buffer, $\mathrm{pH}$ 6.0. The reaction mixture also consisted of 5\% ethanol and $0.10-0.15 \mathrm{mM}$ FAD cofactor. Reactions were quenched by addition of $100 \mathrm{~mL}$ chloroform. Extraction of the lipid components from the reaction mixture was carried out by Bligh-Dyer method similar to $1 \mathrm{~mL}$-scale assays, except the volumes of solvents used for extraction were increased 100-fold and the extraction was carried out in $1000 \mathrm{~mL}$ separatory funnel. Once the bottom chloroform phase was recovered, the solvent was evaporated and the lipid mixture was redissolved in ethyl acetate $(2-4 \mathrm{~mL})$ for purification of HFA product using Silica gel on TLC plates ( $\left.\mathrm{L}^{*} \mathrm{~W}, 20 \mathrm{~cm} * 20 \mathrm{~cm}\right)$. The solvent path was $18 \mathrm{~cm}$ and the development system was chloroform: methanol $(10: 1 \mathrm{v} / \mathrm{v}) .0 .1 \% 2,7-$ Dichlorofluorescein solution in ethanol was used to spray TLC plates. The products on TLC plate 
were visualized under $365 \mathrm{~nm}$ UV light that showed two distinct bands, one for substrate and one for product. The band with lower $\mathrm{R}_{\mathrm{f}}$ value, which belonged to the product, was scraped out of the TLC plates. The product in silica gel powder was extracted with ethyl acetate, which was evaporated under nitrogen flow. Dried sample was dissolved in 1-2 mL of chloroform and extracted twice with Bligh-Dyer method (water was used instead of $1.5 \% \mathrm{KCl}$ ) to remove 2, 7Dichlorofluorescein. Chloroform phase was collected and dried under nitrogen. Amount of dried product was measured for yield calculation. Purity analysis of the products were carried out by GCFID. Detailed description of each preparative scale reaction, conversion percentages, isolated yield values and purity levels are given in Supporting Information (Table S7).

\section{Product Analysis, Identification and Quantification}

Prior to chromatographic analysis, the extracted reaction mixture was derivatized into their trimethylsilyl (TMS) derivatives by the addition of $150 \mu \mathrm{L}$ of $\mathrm{N}, \mathrm{O}-$ Bis(trimethylsilyl)trifluoroacetamide with trimethylchlorosilane (BSTFA + TMCS, 99:1). The silylation reaction was performed at $60{ }^{\circ} \mathrm{C}$ for $30 \mathrm{~min}$. After derivatization, samples were injected in splitless mode (1-2 $\mu$ l) into Scion 436-GC (Bruker, Billerica, MA) system equipped with a flame ionization detector (FID) and a non-polar fused silica capillary SPB-1 column (30 m length x 0,25 mM I.D., 0,25 $\mu \mathrm{M}$ film thickness; Sigma). The oven temperature was set to $220{ }^{\circ} \mathrm{C}$ and it was maintained constant for $60 \mathrm{~min}$ of the total analysis time. Injection temperature was $250{ }^{\circ} \mathrm{C}$ and the column flow was maintained at a constant pressure of 22 Psi throughout the analysis with helium as the carrier gas.

For the separation of closely eluting HFA regioisomers, changes were made to the standard GC method. In the modified method, the injection volume to the GC was typically 0.5 to $1 \mu \mathrm{L}$ and injection was performed in split mode, at a split ratio of 1:20. Initial oven temperature was $90{ }^{\circ} \mathrm{C}$ 
for $1 \mathrm{~min}$ and increased to $170{ }^{\circ} \mathrm{C}$ at a rate of $20^{\circ} \mathrm{C} / \mathrm{min}$. This was immediately followed by a further increase to $220{ }^{\circ} \mathrm{C}$ at a rate of $2{ }^{\circ} \mathrm{C} / \mathrm{min}$ and this temperature was maintained for 16 to 62 min, depending on the chain length of products.

Controls with LA, OA and ricinoleic acid showed that the relative response factors for the TMS derivatives of the substrate FA and its corresponding HFA product are similar (within 5\% deviation; Figure S48) with GC-FID detection under the conditions used, similar to prior observations $^{[19]}$. Thus, quantification of the product was based on the ratio of the peak area of product peak to the total peak areas of both substrate and product. A control sample containing only substrate was used to confirm that substrate and product total peak area are consistent between different measurements. An internal standard (palmitic or heptadecanoic acid) was also added to each sample before extraction, at a concentration of $1 \mathrm{mg} / \mathrm{mL}$, to evaluate consistency of extraction and GC measurements.

Chemical structure identification of HFA products were performed by ${ }^{1} \mathrm{H},{ }^{13} \mathrm{C}$ NMR and by ${ }^{1} \mathrm{H}-$ ${ }^{1} \mathrm{H}$ double-quantum-filtered chemical-shift correlation spectroscopy (DQF-COSY) analysis of purified samples and by GC-MS analysis of TMS derivatives. For NMR analysis, 3-5 mg of samples were dissolved in $\mathrm{CDCl}_{3}$ and spectra were recorded on a Bruker Avance III spectrometer (400 MHz). GC-MS analysis was performed using Thermo Fisher DSQ II quadrupole mass spectrometer equipped with Trace GC Ultra and TriPlus autosampler. Ionization was with electron impact at $70 \mathrm{eV}$ in positive-mode. Ion source temperature was $230^{\circ} \mathrm{C}$ and transfer line was at 250 ${ }^{\circ} \mathrm{C}$. The same capillary column as in GC analysis (SPB-1) was also used for GC-MS analysis with the same temperature program. NMR spectra and GC-MS spectra of all the products identified in this study are given in Supporting Information (Figures S6-S39). 
Absolute configuration of products were determined using Mosher Ester method ${ }^{[20]}$, where methylated HFA products (5-15 mg) were reacted with $(S)-(+)-M T P A$ chloride and $(R)-(-)$-MTPA chloride (see Supporting Information for details). The resulting Mosher esters were dissolved in $0.7 \mathrm{~mL}$ of $\mathrm{CDCl}_{3}$ for ${ }^{1} \mathrm{H} \mathrm{NMR}$ and DQF-COSY analysis. Enantiomeric excess (\% ee) was determined by GC-FID analysis of methylated HFA products by a chiral column (CP-Chirasil Dex CB; 25 m length x 0,25 mM I.D., 0,25 $\mu \mathrm{M}$ film thickness; Agilent).

\section{Microalgae Cultivation}

Two microalgae species, Nannochloropsis gaditana (SAG 2.99) and Pavlova lutheri (SAG 9261) were obtained from Sammlung von Algenkulturen der Universität Göttingen (SAG) culture collection in Germany. The cultivation was carried out in 1-20 L GS Schott bottles in F2P growth medium $^{[21]}$. All reactors were continuously aerated with 5\% carbon dioxide/air mixture under LED illumination with an irradiance of 100-200 $\mu \mathrm{mol}$ photon $\mathrm{m}^{-1} \mathrm{~s}^{-2}$ and 12:12 (h) photoperiod. The cultures were mixed by rotary shaking at $90 \mathrm{rpm}$. Cultivation temperature range was $24 \pm 1{ }^{\circ} \mathrm{C}$ and $\mathrm{pH}$ 7-8. $\mathrm{pH}$ monitoring was performed by Milwaukee MC-122-pH controller (Milwaukee Electronics, Hungary) equipped with a solenoid valve to control $\mathrm{CO}_{2}$ addition. Biomass separation was done by centrifugation at $6500 \times \mathrm{g}$ for $P$. lutheri and $6000 \mathrm{x} g$ for $N$. gaditana for $10 \mathrm{~min}$ followed by freeze-drying. Samples were stored at $-20^{\circ} \mathrm{C}$.

\section{Extraction of FAs from Microalgae Biomass}

Mixture of chloroform:methanol:water (1.25 mL: $2.5 \mathrm{~mL}: 0.45 \mathrm{~mL})$ was added onto $100 \mathrm{mg}$ of dry microalgae biomass. The resuspension was vortexed/shaken for $10 \mathrm{~min}$ followed by $15 \mathrm{~min}$ of sonication in a bath sonicator (at $50 \mathrm{~Hz})$. After a further addition of chloroform:methanol (1.25 $\mathrm{mL}: 1.25 \mathrm{~mL}$ ), the mixture was vortexed/shaken for 5 more minutes. This was followed by centrifugation at $3,000 \mathrm{~g}$ for $15 \mathrm{~min}$. The bottom chloroform layer (including total lipids) was 
recovered and transferred into new tubes before being completely dried under a flow of nitrogen. In order to achieve hydrolysis of triglycerides, $1 \mathrm{~mL}$ of $0.5 \mathrm{M} \mathrm{KOH}$ in methanol was added and the resulting mixture was incubated at $100{ }^{\circ} \mathrm{C}$ for $10 \mathrm{~min}$. Afterwards, methanol:water:hexane (1.3 $\mathrm{mL}: 2 \mathrm{~mL}: 4 \mathrm{~mL}$ ) was added and the mixture was vortexed vigorously for extraction of the FAs in $\mathrm{K}^{+}$salt form. After centrifugation, the bottom water layer was collected and acidified using $6 \mathrm{~N}$ $\mathrm{HCl}$ to bring $\mathrm{pH}$ into 3-5 range. This was followed by addition of $4 \mathrm{~mL}$ of hexane and extraction of hexane phase. Once the hexane was evaporated, the amount of dry FAs were determined by weighing and proper amount of ethanol was added to achieve a stock concentration of $20 \mathrm{mg} / \mathrm{mL}$ of total FAs. Total lipid yield from N. gaditana was $11.5 \%$ and from P. lutheri was $32.3 \%$. Total FA content was $33.5 \%$ and $17.9 \%$ of the total lipids for $N$. gaditana and P. lutheri, respectively. FA composition analysis was performed by GC analysis of TMS derivatives of the extracted FAs. Identification of each FA was performed by GC-MS.

\section{Structure Analysis, Homology Modelling and Substrate Docking Studies}

Crystal structure images were produced using the UCSF Chimera package from the Computer Graphics Laboratory, University of California, San Francisco (supported by NIH P41 RR01081) ${ }^{[22]}$. Homology modelling was performed using Modeller 9.21 program $^{[23]}$. Chain A of OhyA (pdb id: 4uirA) was used as a template, where FAD and substrate analog was kept as rigid bodies during homology modelling run. All six models produced for each protein were of good quality and had a GA341 score of 1.0. The best model was decided according to molpdf and DOPE scores. The stereochemical quality of the homology models were analyzed by the Ramachandran Plot analysis server RAMPAGE ${ }^{[24]}$, which showed less than $2.5 \%$ of residues in outlier region for all models. Substrate docking was performed using AutoDock Vina ${ }^{[25]}$, by implementation through Chimera. Protein structures (with P6G substrate analog deleted) and FA substrates were prepared 
by DockPrep tool of Chimera. Analysis of the docking modes were performed through ViewDock tool, which listed 10 docking modes according to their energy scores. Best docking model was decided by a combination of criteria; visual inspection (correct orientation of the carboxylate and omega ends), observed regioselectivity, reasonable distances to catalytic residues and energy scores. More details are given in Supporting Information.

\section{RESULTS}

Design of FA-HY2 Mutants. In order to reveal the basis of substrate specificity and regioselectivity difference between FA-HY1 and FA-HY2, a comparative analysis of the activesite (within $\sim 5 \AA$ of the substrate and FAD) of the two enzymes was performed. This analysis was based on the FAD and substrate analog (hexaethylene glycol; P6G) bound structure of the closely related homolog Ohy ${ }^{[17]}$ (pdb id: 4uir; 62\% similarity to FA-HY1; Figure 1). Three amino acids attracted our attention in this analysis. Two of these residues were equivalent to the amino acids that were shown to interact with the carboxylate end of the FA substrate in an OA docking model of OhyA structure ${ }^{[17]}$ (T436 and N438 in OhyA; Figure 1). Apparently, while OhyA and FA-HY2 have identical (T436 vs. T391 in FA-HY2) or similar residues (N438 vs. H393 in FA-HY2) at these corresponding positions, FA-HY1 had less bulky serine residues at both positions. It was previously demonstrated that mutation of these two residues to alanine abolished the activity significantly in Ohy $\mathrm{A}^{[17]}$. Moreover, an isoleucine (I423 in OhyA) was identified, which is also an isoleucine in FA-HY2 (I378), but a proline in FA-HY1 (P380) at corresponding position (Figure 1A). This residue is in close proximity to the carboxylate-anchoring residues of T436 and N438 (OhyA numbering), as well as to the substrate analogue. To evaluate the effect of the three amino acids on substrate specificity and regiodiversity, these residues on FA-HY2 were mutated to the corresponding residues on FA-HY1. Single mutations of T391S (T391S-FA-HY2) and H393S 
(H393S-FA-HY2) were designed as well as a double mutant (DM-FA-HY2;T391S/H393S) and a triple mutant (TM-FA-HY2; T391S/H393S/I378P). H393Q mutant (H393Q-FA-HY2) was also generated to further evaluate the plasticity of the residue at this position (Table S2).
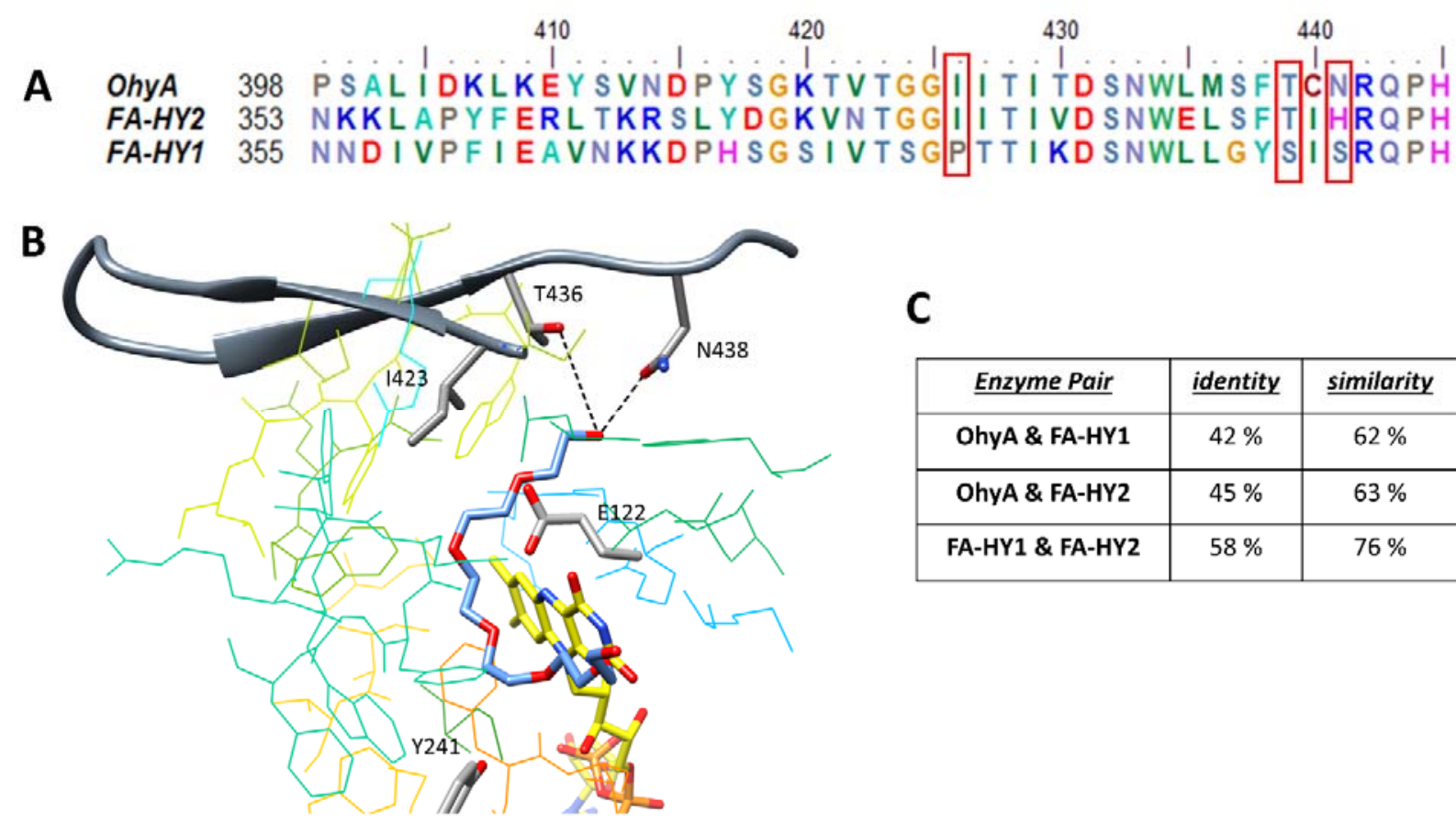

C

\begin{tabular}{|c|c|c|}
\hline Enzyme Pair & identity & similarity \\
\hline OhyA \& FA-HY1 & $42 \%$ & $62 \%$ \\
\hline OhyA \& FA-HY2 & $45 \%$ & $63 \%$ \\
\hline FA-HY1 \& FA-HY2 & $58 \%$ & $76 \%$ \\
\hline
\end{tabular}

Figure 1. A) Partial sequence alignment of OhyA, FA-HY1 and FA-HY2 highlighting the residues (in red boxes) mutated in this study. B) Location of the residues in the active site of OhyA (pdb id: 4uir) with respect to the substrate analogue (P6G; ethylene units represented in blue color) and catalytic residues of E122 and Y241. FAD is shown in yellow. Ribbon representation is shown only for mutated residues and their nearby neighbours. T436 and N438 reside at the edge of a small loop region between two beta-sheets. I423 resides at the junction of a loop and beta-sheet. C) Percent identity and similarity levels between OhyA, FA-HY1 and FA-HY2.

Analysis of Substrate Specificity and Regioselectivity. Activities of FA-HY1, FA-HY2 and mutant enzymes were screened towards 12 FA substrates with various chain lengths and different degrees of unsaturation (Scheme 2). The results are presented in Figure 2, Table 1 and Tables S10S12. Both wild-type and mutant hydratases displayed varying levels of hydration activity towards 10 out of 12 FAs. For trans-vaccenic acid and LA methyl ester, no activity was observed with any 
of the enzymes, indicating essential requirement of cis-conformation and free carboxylate group of substrates, consistent with earlier reports ${ }^{[18]}$. Confirmation for the identity of the products have been carried out by GC-MS analysis of TMS derivatives and by ${ }^{1} \mathrm{H}$ and ${ }^{13} \mathrm{C}$ NMR of purified products (Figures S6-S39).

Scheme 2. Structures and names of all fatty acid substrates used in this study.

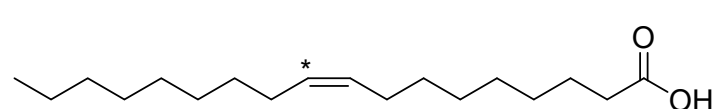

oleic acid (cis-9-octadecanoic acid)

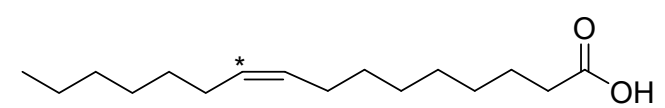

palmitoleic acid (cis-9-hexadecanoic acid)

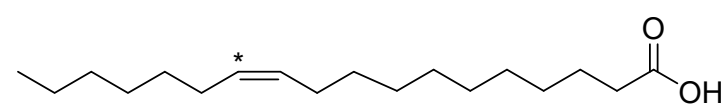

cis-vaccenic acid (cis-11-octadecanoic acid)

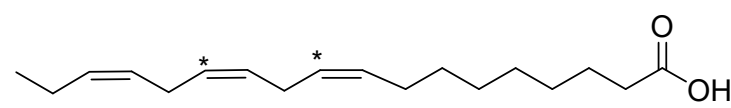

alpha-linolenic acid (cis-9,cis-12,cis-15-octadecatrienoic acid)

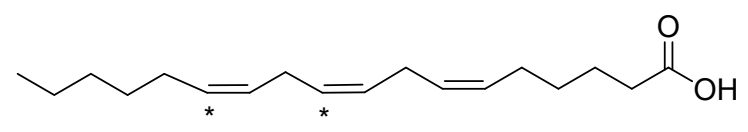

gamma-linolenic acid (cis-6,cis-9,cis-12-octadecatrienoic acid)

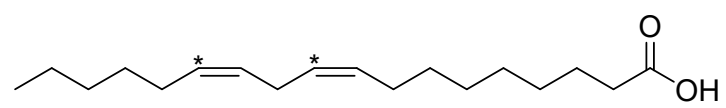

linoleic acid (cis-9,cis-12-octadecadienoic acid)
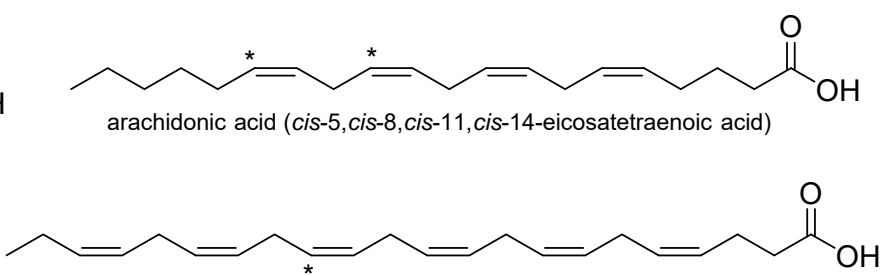

DHA (cis-4,cis-7,cis-10,cis-13,cis-16,cis-19-docosahexaenoic acid)

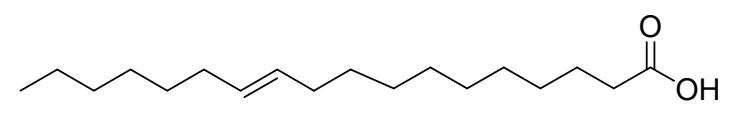

trans-vaccenic acid (trans-11-octadecanoic acid)

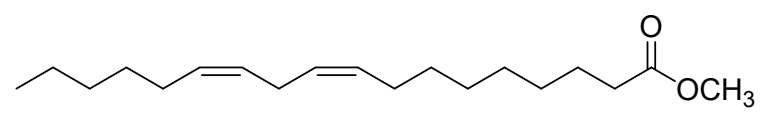

Linoleic acid methyl ester (methyl cis-9,cis-12-octadecadienoate)

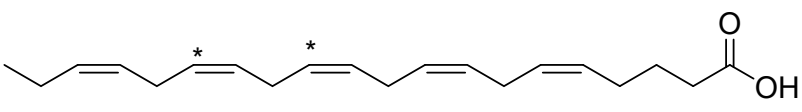

EPA (cis-5,cis-8,cis-11,cis-14,cis-17-eicosapentaenoic acid)

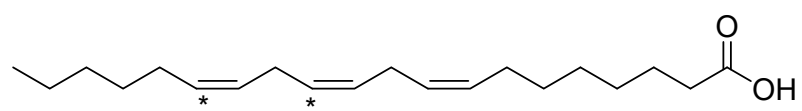

ETA (cis-8,cis-11,cis-14-eicosatrienoic acid)

*position of hydroxylation for the products observed.

Comparison of the activities of wild-type FA-HY1 and wild-type FA-HY2 towards OA and cisvaccenic acid reveals the significance of double bond position of FA. Both OA and cis-vaccenic acid are $\mathrm{C} 18: 1$ FAs with a single double bond in cis configuration; at $\Delta 9$ position for OA and at $\Delta 11$ position for cis-vaccenic acid. FA-HY1 converted over $90 \%$ of cis-vaccenic acid to $12-\mathrm{OH}$ product, but had almost 20 -fold lower activity towards OA (less than $5 \%$ conversion). For FA- 
HY2, the situation was completely reversed; over $90 \%$ conversion with OA, but no detectable activity towards cis-vaccenic acid (Figure 2A, Table S10).

Mutant enzymes showed remarkable shift from FA-HY2 towards FA-HY1 (Figure 2, S1 and Table 1). Our results demonstrate significant regioselectivity shift in the case of LA. TM-FA-HY2 can now convert about $60 \%$ of substrate into $13-\mathrm{OH}$ product and only $8 \%$ into $10-\mathrm{OH}$, whereas FA-HY2 almost exclusively produces 10-OH and FA-HY1 exclusively 13-OH (Figure 2B). This indicates an almost complete reversal of regioselectivity against LA for TM-FA-HY2, shifting ratio of the regioisomers of HFA products (10-OH:13-OH) from 99:1 to 12:88 (Table 1). DM-FA-HY2 showed almost equal split (54:46) between $10-\mathrm{OH}$ and $13-\mathrm{OH}$ products. Besides, total turnover numbers were also higher for both DM-FA-HY2 and TM-FA-HY2 compared to FA-HY2, with $50 \%$ and $80 \%$ increases respectively. Both mutants also had higher total turnover numbers than FA-HY1 (Table 1).

In the case of palmitoleic acid (POA), three mutations on FA-HY2 decreased the activity from $90 \%$ to $5 \%$ conversion, bringing down TM-FA-HY2 activity to almost same level as FA-HY1 ( $4.2 \%$ conversion) (Figure 2A). Both regioselectivity and total activity shift were observed in the case of AA, where TM-FA-HY2 can carry out $\sim 50 \%$ total conversion compared to only $2 \%$ conversion by FA-HY2 (Figure 2D and Tables S11-S12). Regioselectivity shift was significant for DM-FA-HY2 with AA, exhibiting a 47:53 regioisomer ratio (12-OH:15-OH) (Table S12). While FA-HY2 can only produce 12-OH product from AA, both DM-FA-HY2 and TM-FA-HY2 exhibit around $5 \%$ conversion to $15-\mathrm{OH}$, which is the major product of FA-HY1. TM-FA-HY2 exhibited about 15-fold higher conversion of EPA to 12-OH product with respect to FA-HY2 and 5-fold higher conversion with respect to FA-HY1 under identical conditions. While FA-HY2 is totally inactive towards DHA, histidine mutants as well as DM-FA-HY2 and TM-FA-HY2 had the same 
level of activity as FA-HY1 ( 2-3\%). Hence, just two to three mutations (DM-FA-HY2 and TMFA-HY2) were sufficient to convert FA-HY2 to an FA-HY1-like enzyme, without sacrificing much of their original activity against the conventional hydratase substrates of OA, alpha-linolenic and gamma-linolenic acids (Figure 2, Tables S10-S11). Among all the variants, only I378P had very low activities or was totally inactive. However, when this mutation is coupled with T391S and H393S mutations, activities are rescued and even increased for some of the substrates. It is also interesting to note that H393Q mutation did not impair the activity of FA-HY2.

Furthermore, di-hydroxy products 10,13-dihydroxy octadecanoic acid, was also observed for LA when H393S-FA-HY2, DM-FA-HY2 or TM-FA-HY2 are used, although none has been detected for wild-type enzymes (Figure 2B, S1 and Table S11). Overall conversion levels of LA by these three mutant enzymes are about 50\% higher compared to wild-type enzymes.

Table 1. Impact of double and triple mutations on activity and regioselectivity towards linoleic $\operatorname{acid}^{\mathrm{a}}$.

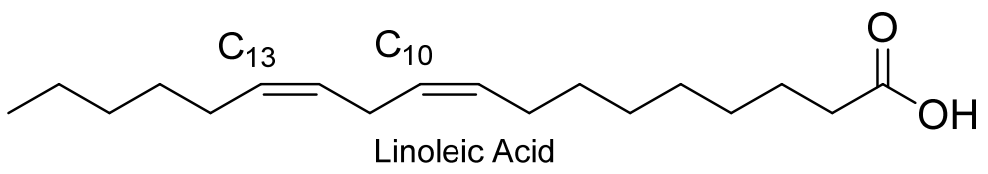

\begin{tabular}{|c|c|c|c|c|c|}
\hline Enzyme & $\begin{array}{l}10-\mathrm{OH} \text { ODA }{ }^{[\mathrm{b}]} \\
(\% \text { conversion })\end{array}$ & $\begin{array}{l}13-\mathrm{OH} \mathrm{ODA}{ }^{[\mathrm{b}]} \\
(\% \text { conversion})\end{array}$ & $\begin{array}{c}\text { 10,13-di- } \\
\text { OHODA }^{[\mathrm{b}]} \\
(\% \text { conversion })\end{array}$ & $\begin{array}{c}\text { 10-OH/13-OH } \\
\text { ratio }^{[\mathrm{c}]}\end{array}$ & $\mathrm{TTN}^{[\mathrm{d}]}$ \\
\hline FA-HY1 & 0 & $47.9 \pm 1.7$ & 0 & $0: 100$ & 190 \\
\hline FA-HY2 & $40.9 \pm 6.8$ & $0.5 \pm 0.4$ & 0 & $99: 1$ & 165 \\
\hline $\begin{array}{c}\text { DM-FA- } \\
\text { HY2 }\end{array}$ & $30.5 \pm 1.8$ & $26.3 \pm 0.4$ & $7.3 \pm 0.5$ & $54: 46$ & 254 \\
\hline $\begin{array}{c}\text { TM-FA- } \\
\text { HY2 }\end{array}$ & $7.9 \pm 0.9$ & $58.7 \pm 2.4$ & $8.4 \pm 1.9$ & $12: 88$ & 298 \\
\hline
\end{tabular}



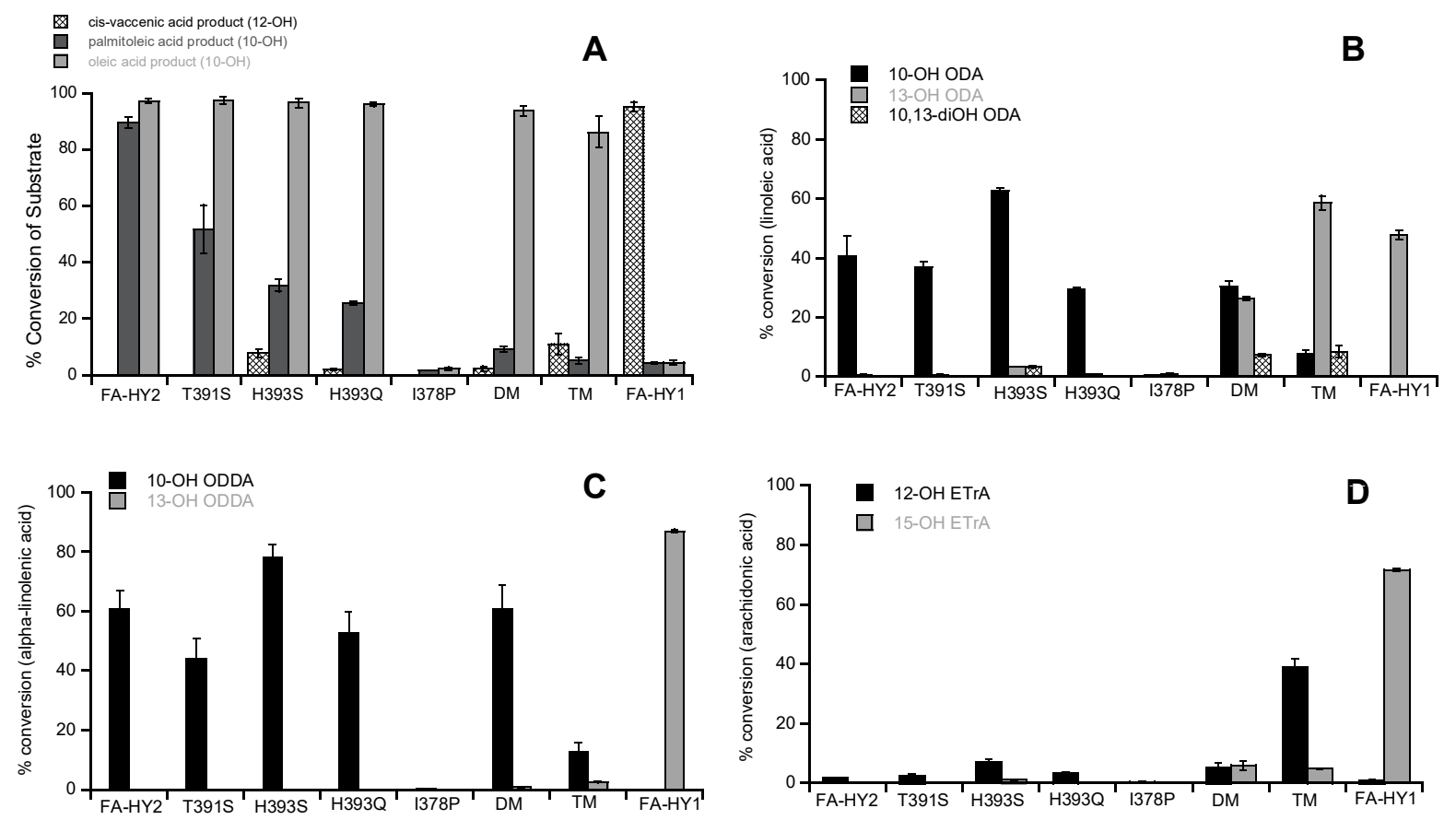

Figure 2. A) Conversion levels of wild-type and mutant fatty acid hydratases for monounsaturated FA substrates. Error bars indicate standard error for mean value of at least two separate experiments. Conversion levels of wild-type and mutant fatty acid hydratases; B) for conversion of linoleic acid substrate into products of 10-OH ODA (10-hydroxycis-12-octadecenoic acid), 13-OH ODA (13-hydroxy-cis-9-octadecenoic acid) and 10,13-diOH ODA (10,13dihydroxy octadecanoic acid); C) for conversion of alpha-linolenic acid substrate into products of 10-OH ODDA (10hydroxy-cis-12,cis-15-octadecadienoic acid), 13-OH ODDA (13-hydroxy-cis-9,cis-15-octadecadienoic acid); D) for conversion of arachidonic acid substrate into products of 12-OH ETrA (12-hydroxy-cis-5,cis-8,cis-14-eicosatrienoic acid), 15-OH ETrA (15-hydroxy-cis-5,cis-8,cis-11-eicosatrienoic acid). Error bars indicate standard error for mean value of at least two separate experiments.

Preparative Scale Reactions and Structural Identification. In order to evaluate conversion levels at preparative scale and to isolate and determine structure of new HFA products by NMR, select reactions were set up in $100 \mathrm{~mL}$ scale with a quantity of 50-100 $\mathrm{mg}(0.15-0.35 \mathrm{mmol}$ scale $)$ of substrates and with a concentration of $0.1 \mathrm{mg} / \mathrm{mL}-0.5 \mathrm{mg} / \mathrm{mL}(1.5-9.0 \mu \mathrm{M})$ of enzymes. 
Reactions were carried out for $\sim 24$ hours at $37^{\circ} \mathrm{C}$. TM-FA-HY2 variant exhibited conversion levels of $92 \%$ for ETA as substrate and $67 \%$ for EPA as substrate. After extraction and purification by preparative TLC, 12-OH product of ETA ((S)-12-hydroxy-cis-8, cis-14-eicosadienoic acid) was isolated with a 53\% yield and 12-OH product of EPA ((S)-12-hydroxy-cis-5,cis-8,cis-14,cis-17eicosatetraenoic acid) was isolated with a yield of $31 \%$. Both products were in (S)-configuration with an enantiomeric excess ( $\%$ ee) of at least $95 \%$, as determined by chiral GC analysis and by Mosher Ester method (Figures S40, S42). Preparative scale reactions were also carried out using TM-FA-HY2 with AA and OA as substrates and using H393S with LA as substrate, in addition to conversions with FA-HY1 (AA and LA) and with FA-HY2 (LA and OA). Varying conversion levels were obtained, from 5\% to 99\% (Table S7). 13-OH LA product from FA-HY1 reaction and 10-OH LA product from H393S reaction were isolated in pure form with yields of $29 \%$ and $13 \%$, respectively, and with high enantiopurities ( $>95 \%$ ee). While $10-\mathrm{OH}$ LA product was in $(S)$ configuration, 13-OH LA was determined to be in $(R)$-configuration due to change in Cahn-IngoldPrelog priority rules upon double bond positions. Majority of the purified HFA products had a purity of $>95 \%$ as determined by GC-FID analysis. Structural analysis of purified samples were confirmed by NMR $\left({ }^{1} \mathrm{H}\right.$ and ${ }^{13} \mathrm{C}$ ) analysis (Figures S7-S39). Absolute configuration of new products were determined by Mosher Ester method (Tables S2-S6; Figures S40-S46). Details of the conversion and yield analysis of all reactions are given in Table S7.

Steady-state Kinetics. In order to gain more insight into the activity differences between wildtype and mutant enzymes, $k_{\mathrm{cat}}$ and apparent $K_{\mathrm{m}}$ values towards selected substrates were measured (Table 2, Figures S2-S5). FA-HY1 is almost 4-orders of magnitude catalytically more efficient (based on $k_{\text {cat }} / K_{\mathrm{m}}$ value) compared to FA-HY2 with LA as substrate (12900 vs. 2.6). This is particularly due to the significant difference in $k_{\text {cat }}$ values $\left(340 \mathrm{~min}^{-1}\right.$ vs. $\left.0.36 \mathrm{~min}^{-1}\right) . K_{\mathrm{m}}$ values are 
comparable within error percentage indicating that both enzymes can bind LA with similar affinity. Moreover, double and triple mutations on FA-HY2 increased $k_{\text {cat }}$ significantly (about 10 to 20 -fold) for LA with respect to FA-HY2, which should also be responsible for the increased overall yield (vide supra). However, no significant difference was observed for $K_{\mathrm{m}}$, again suggesting that binding affinity is not affected.

Table 2. Steady-state kinetic parameters ${ }^{[\mathrm{a}]}$ for FA-HY1, FA-HY2 and selected mutants.

\begin{tabular}{|c|c|c|c|c|c|}
\hline Enzyme & Substrate & Product $^{[b, c]}$ & $k_{\text {cat }}(1 /$ min $)$ & $K_{\mathrm{m}}(\mathrm{mM})$ & $\begin{array}{c}k_{\text {cat }} / K_{\mathrm{m}} \\
(1 / \mathrm{mM} \cdot \mathbf{m i n})\end{array}$ \\
\hline FA-HY1 & LA & 13-OH ODA & $340 \pm 41$ & $\begin{array}{c}0.026 \pm \\
0.008\end{array}$ & $12900 \pm 2300$ \\
\hline FA-HY2 & LA & 10-OH ODA & $0.36 \pm 0.06$ & $\begin{array}{c}0.139 \pm \\
0.078\end{array}$ & $2.6 \pm 1.0$ \\
\hline $\begin{array}{l}\text { DM-FA- } \\
\text { HY2 }\end{array}$ & LA & $\begin{array}{c}(13-\mathrm{OH} \text { ODA }+10 \\
\text { OH ODA })\end{array}$ & $3.04 \pm 0.34$ & $\begin{array}{c}0.057 \pm \\
0.018\end{array}$ & $54.0 \pm 13.0$ \\
\hline $\begin{array}{l}\text { TM-FA- } \\
\text { HY2 }\end{array}$ & LA & $\begin{array}{c}(13-\mathrm{OH} \text { ODA }+10 \\
\text { OH ODA })\end{array}$ & $5.81 \pm 0.71$ & $\begin{array}{c}0.036 \pm \\
0.015\end{array}$ & $160 \pm 50$ \\
\hline FA-HY1 & OA & 10-OH SA & $\begin{array}{c}0.026 \pm \\
0.001\end{array}$ & $\begin{array}{c}0.028 \pm \\
0.009\end{array}$ & $0.93 \pm 0.25$ \\
\hline FA-HY2 & OA & $10-\mathrm{OH} \mathrm{SA}$ & $7.12 \pm 0.65$ & $\begin{array}{c}0.020 \pm \\
0.008\end{array}$ & $360 \pm 110$ \\
\hline $\begin{array}{l}\text { TM-FA- } \\
\text { HY2 }\end{array}$ & OA & $10-\mathrm{OH} \mathrm{SA}$ & $0.69 \pm 0.07$ & $\begin{array}{c}0.016 \pm \\
0.007\end{array}$ & $43.0 \pm 15.0$ \\
\hline FA-HY1 & POA & 10-OH PA & $\begin{array}{c}0.061 \pm \\
0.007\end{array}$ & $\begin{array}{c}0.376 \pm \\
0.095\end{array}$ & $0.16 \pm 0.02$ \\
\hline FA-HY2 & POA & 10-OH PA & $0.89 \pm 0.10$ & $\begin{array}{c}0.046 \pm \\
0.017\end{array}$ & $19.0 \pm 6.0$ \\
\hline $\begin{array}{l}\text { DM-FA- } \\
\text { HY2 }\end{array}$ & POA & 10-OH PA & $\begin{array}{c}0.063 \pm \\
0.003\end{array}$ & $\begin{array}{c}0.030 \pm \\
0.007\end{array}$ & $2.1 \pm 0.4$ \\
\hline $\begin{array}{l}\text { TM-FA- } \\
\text { HY2 }\end{array}$ & POA & 10-OH PA & $0.017 \pm 0.001$ & $\begin{array}{c}0.039 \pm \\
0.007\end{array}$ & $0.42 \pm 0.07$ \\
\hline FA-HY1 & AA & $\begin{array}{c}(12-\mathrm{OH} \text { ETrA+15 } \\
\text { OH ETrA })\end{array}$ & $5.7 \pm 0.6$ & $\begin{array}{c}0.076 \pm \\
0.023\end{array}$ & $75.0 \pm 17.0$ \\
\hline $\begin{array}{l}\text { TM-FA- } \\
\text { HY2 }\end{array}$ & AA & $\begin{array}{c}(12-\mathrm{OH} \text { ETrA+15 } \\
\text { OH ETrA })\end{array}$ & $0.89 \pm 0.06$ & $\begin{array}{c}0.020 \pm \\
0.007\end{array}$ & $45.0 \pm 13.0$ \\
\hline
\end{tabular}


${ }^{[\mathrm{a}]}$ Michaelis-Menten plots are given in Supporting Information. ${ }^{[\mathrm{b}]}$ Product abbreviations: 10-OH ODA (10-hydroxycis-12-octadecenoic acid); 13-OH ODA (13-hydroxy-cis-9-octadecenoic acid); 10-OH SA (10-hydroxy-stearic acid); 10-OH PA (10-hydroxy palmitic acid); 12-OH ETrA (12-hydroxy-cis-5,cis-8,cis-14-eicosatrienoic acid); 15-OH ETrA (15-hydroxy-cis-5,cis-8,cis-11-eicosatrienoic acid). ${ }^{[\mathrm{c}]}$ In the case where two products are formed, the total amount of products was taken into account for kinetic analysis. It was observed that the kinetics for the formation of each product separately were almost identical.

Although long-time conversion levels of both FA-HY2 and TM-FA-HY2 are close to $100 \%$ for $\mathrm{OA}, k_{\mathrm{cat}} / K_{\mathrm{m}}$ for TM-FA-HY2 is about an order of magnitude less compared to that of FA-HY2 and in a range that is almost half-way between catalytic efficiencies of FA-HY1 and FA-HY2 (Table 2). Similar difference was also observed for POA when FA-HY2 is compared to both DM-FAHY2 and TM-FA-HY2. As for AA, FA-HY1 exhibits higher $k_{\text {cat }}$ and $K_{\mathrm{m}}$ values compared to TMFA-HY2. However, catalytic efficiencies are similar (75 vs. 45 ).

Overall, the effect of mutations is significantly reflected on $k_{\mathrm{cat}}$, but not on $K_{\mathrm{m}}$. All measured $K_{\mathrm{m}}$ values for mutant enzymes are within $\sim 2$-fold range of $K_{\mathrm{m}}$ values of FA-HY2 for each respective substrate. However, changes in $k_{\text {cat }}$ values, either increase or decrease, are almost an order of magnitude when DM-FA-HY2 and TM-FA-HY2 are compared to FA-HY2 for all the substrates tested.

Substrate Docking Studies. For a better understanding of the structural basis of substrate specificity and regioselectivity of hydratases, binding modes of LA with respect to carboxylate anchoring and catalytic residues (E82 and Y200 in FA-HY2) were compared. Docking investigation was performed on the homology models of FA-HY1, FA-HY2 and TM-FA-HY2, which were based on the structure of OhyA as the template (Figure 3A). OhyA is the only structure available to date with both FAD and a substrate analog (hexaethylene glycol) bound and is most relevant to catalysis ${ }^{[17]}$. According to the mechanism proposed based on this structure, Y241 (Y200 in FA-HY2) protonates the double bond and E122 (E82 in FA-HY2) activates a water molecule to attack the resulting carbocation at $\mathrm{C} 10$ position $^{[17]}$ (Scheme 3). This mechanism necessitates that 
Y241 and E122 are correctly positioned with respect to the double bond for efficient hydration. In addition, our results indicate that the size of the residue at the position corresponding to N438 in OhyA has a profound effect on substrate specificity and regioselectivity (vide supra).

Scheme 3. Role of catalytic residues in the mechanism of fatty acid hydratase

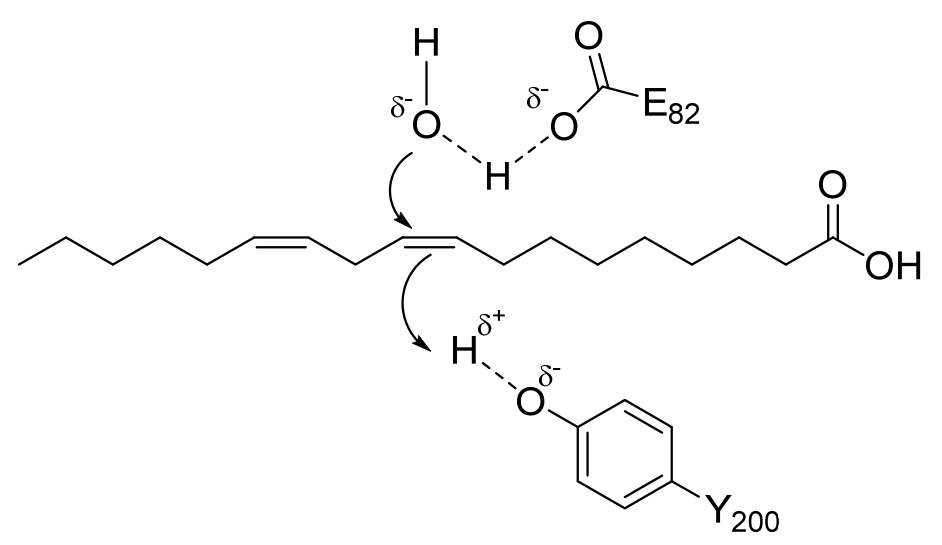

When LA is docked into OhyA structure, carboxylate oxygen has the closest distances of $4.8 \AA$ and $4.7 \AA$ from T436 and N438, respectively, in energetically most favorable binding mode (Figure 3B). In this binding mode, $\mathrm{Y} 241$ is positioned closer to $\Delta 9$ double bond than the $\Delta 12$ double bond (3.5 $\AA$ vs. $5.4 \AA$ ). Unlike E122, which interacts with the double bond indirectly through a water molecule, Y241 should directly protonate the double bond for activation. Thus, a shorter distance of $3.5 \AA$ is in agreement with production of exclusively 10-OH ODA with LA in the case of Ohy ${ }^{[17]}$. A similar pattern is also observed for the docking model of LA into FA-HY2 homology model (Figure 3D), which also almost produces exclusively 10-OH product. In the most plausible binding mode, the positioning of Y 200 is closer to $\Delta 9$ double bond than $\Delta 12$ double bond (4.2 $\AA$ to $\mathrm{C} 10$ vs. $6.1 \AA$ to $\mathrm{C} 13$; Figure $3 \mathrm{D}$ ), consistent with hydration at $\Delta 9$ double bond. However, for FA-HY1 homology model, most plausible binding mode positions Y202 with a closer distance to $\Delta 12$ double bond compared to $\Delta 9$ double bond (6.6 $\AA$ to $\mathrm{C} 10$ vs. $4.0 \AA$ to $\mathrm{C} 13$; Figure $3 \mathrm{C}$ ). Such a binding mode is in agreement with production of 13-OH ODA product due to efficient hydration 
of $\Delta 12$ double bond, as observed experimentally. It is clearly seen from docking models in Figure 3 that smaller serine residues (S393 and S395) in FA-HY1 enables shifting of LA deeper into the carboxylate end of the substrate channel, thus aligning $\Delta 12$ double bond closer to catalytic residues, especially to Y202, consistent with formation of $13-\mathrm{OH}$ ODA as the only product. Size and orientation of P380 residue in FA-HY1 (compared to I378 in FA-HY2) seems to help orienting the FA chain towards S395, by orienting the hydrophobic carbon chain.

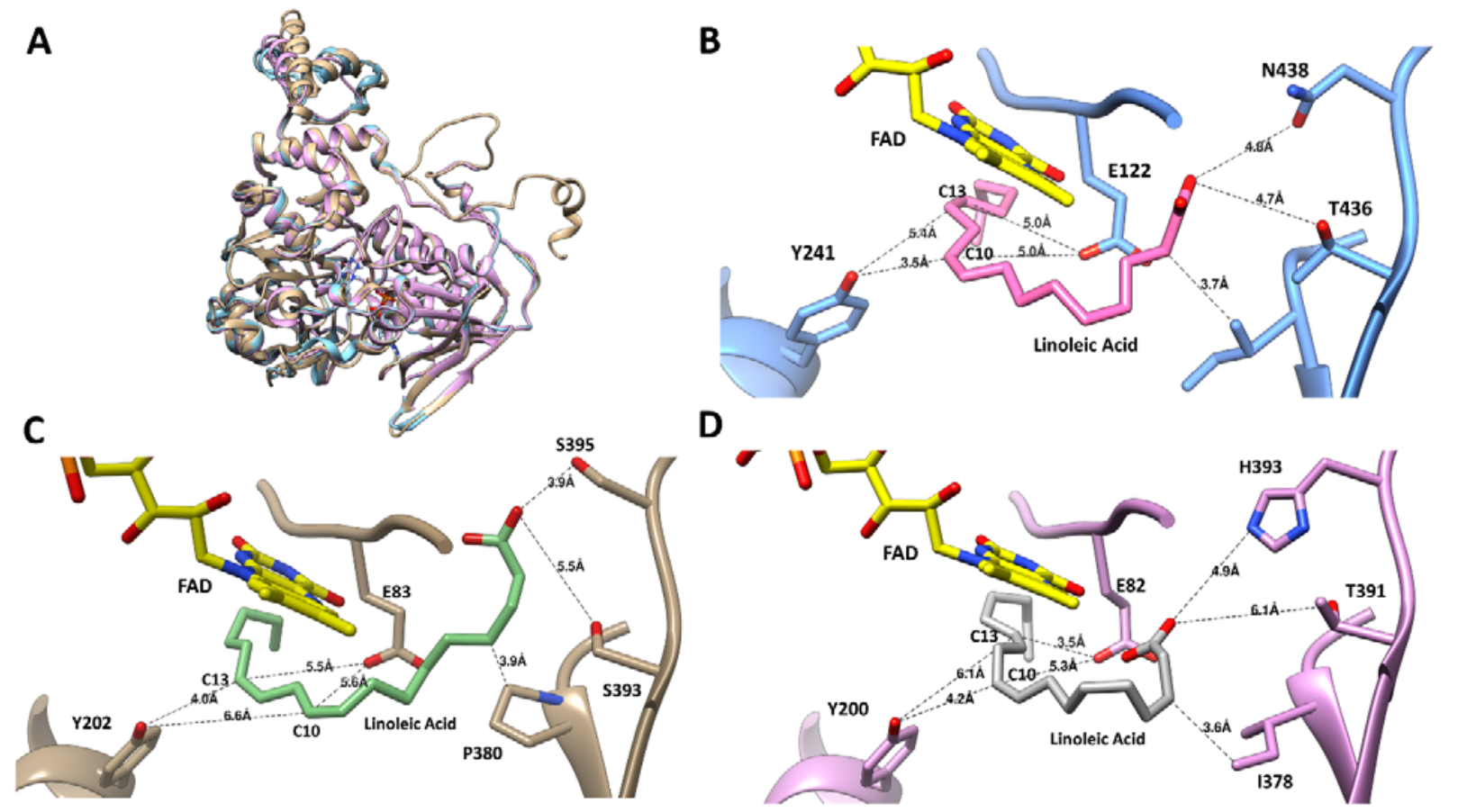

Figure 3. A) Overlaid ribbon representations of the structure of chain A of OhyA (light brown) with homology models of FA-HY1 (light blue) and FA-HY2 (pink). FAD is shown in stick representation. Docking models are shown for the best reasonable binding mode of LA into; B) OhyA active-site; C) into active site of FA-HY1 homology model; D) into active site of FA-HY2 homology model.

Docking of LA into the homology model of TM-FA-HY2 variant exhibited a more similar placement and orientation of LA in the active site to that of FA-HY1 (Figure 4A and 4B). This is consistent with TM-FA-HY2 generating 13-OH LA as the major product. In fact, among the 
binding modes generated by the docking program, one more plausible binding mode can also be found, which aligns Y200 better with $\Delta 9$ double bond (4.4 $\AA$ to $\mathrm{C} 10)$ while still keeping it close to $\Delta 12$ double bond, with a distance of $5.0 \AA$ (Figure 4C). Such a binding mode will explain formation of $10-\mathrm{OH}$ and $10,13-\mathrm{diOH}$ as the minor products. This suggests that TM-FA-HY2 might be attaining both binding modes.

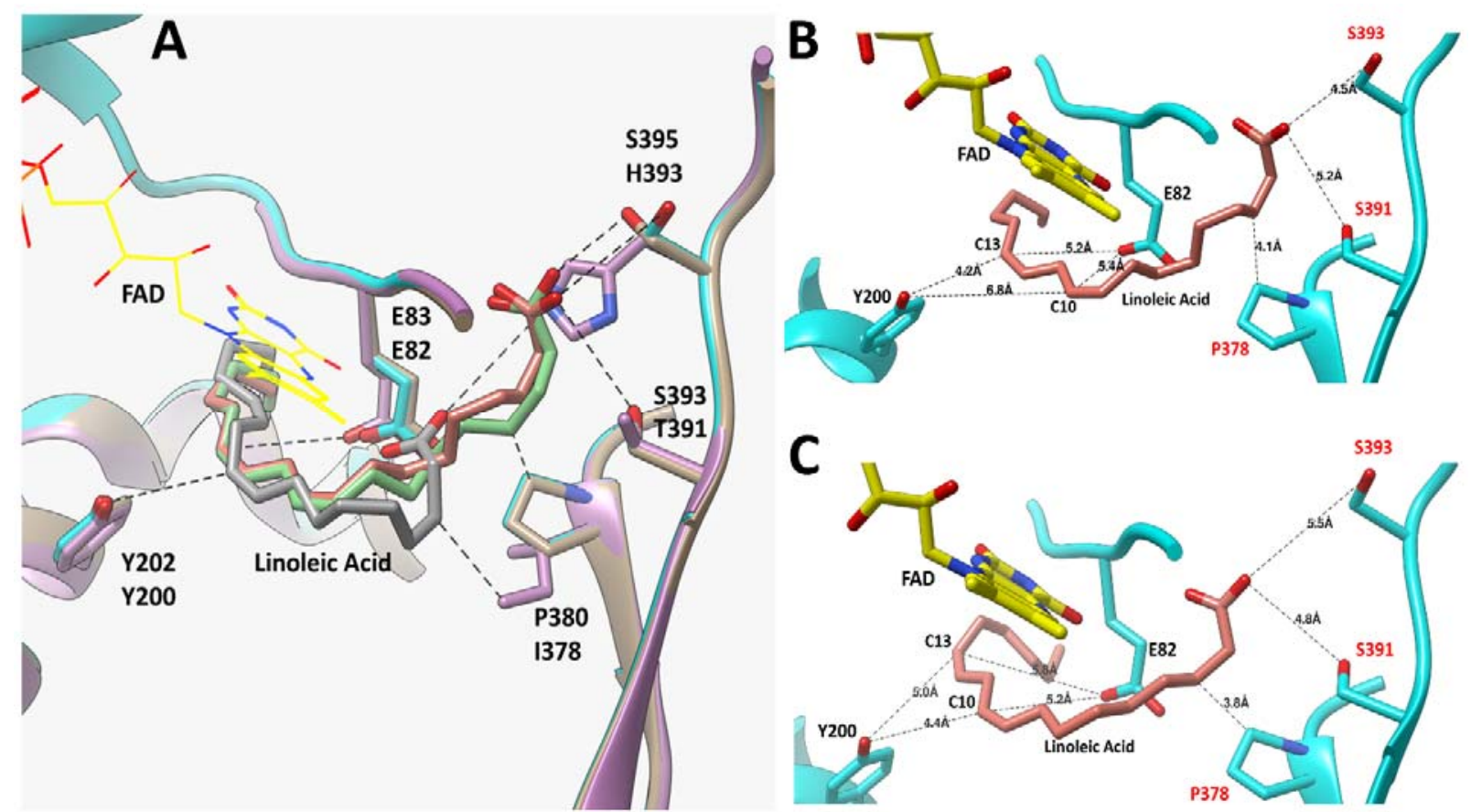

Figure 4. A) Overlaid active site structures of FA-HY1, FA-HY2 and TM-FA-HY2 homology models. For TM-FAHY2, the docking model in Figure 4B is shown. B) Docking model showing LA in the active site of TM-FA-HY2 homology model in the best reasonable binding mode for C13 hydroxylation. C) Docking model showing LA in the active site of TM-FA-HY2 homology model in the best reasonable binding mode for C10 hydroxylation.

Conversion of Microalgae FA into HFAs by Hydratases. Microalgae has great potential as a sustainable source of various FAs ${ }^{[26]}$. Different microalgae species can accumulate a diverse range of saturated and unsaturated long-chain FAs (C14-C22 mainly) that can reach up to 5-15\% of their dry biomass depending on cultivation conditions ${ }^{[27]}$. Nannochloropsis gaditana and Pavlova 
lutheri biomass were used to show the proof-of-concept for sustainable conversion of microalgae FAs into HFAs by hydratases. $N$. gaditana has high content of EPA and POA as well as lower levels of AA, DHA and C18 unsaturated FAs. P. lutheri builds up significant amounts of EPA, DHA and POA (Figure 5A, Tables S13-S14).

Assays were carried out similar to the long-time conversion assays (vide supra). However, instead of using pure FA solutions as substrates, total FA hydrolysate of the lipids extracted from microalgae biomass were used. The results show that wild-type and mutant enzymes can produce HFAs from FAs of microalgae extract almost as efficiently as from pure FA substrates and in some cases even with better yields (Figure 5, Tables S15-S16). Moreover, regioselectivity patterns of the enzymes are retained. There are some noticeable differences for the conversion of some FAs. For example, while POA conversion is about 5\% with FA-HY1 and TM-FA-HY2 using pure substrate, the conversion level increased to $35-50 \%$ with microalgae extracts (Figure 5B). However, conversion of OA and POA with FA-HY2 were decreased from $\sim 90-95 \%$ with pure substrates to $20-45 \%$ with microalgae. GC chromatograms of FA-HY2 reaction exhibited an additional peak that elutes close to OA peak ( $\sim 60 \%$ of the starting substrate amount). According to GC-MS analysis, this peak can be attributed to trans-isomer of the substrate (trans-10-octadecenoic acid), as shown previously for $\mathrm{LA}^{[18 \mathrm{a}]}$. Although this peak was also observed with pure OA at much lower levels (less than 5\%), the extent of the reverse reaction generating trans-isomer is higher with microalgae.

Another significant difference is the increased conversion levels of EPA from microalgae extract in comparison to pure EPA under identical assay conditions. All enzymes, except FA-HY2, became much more efficient for the conversion of EPA to 12-OH product (Figure 5D). When P. lutheri extract (which contains $\sim 22 \%$ of EPA) was used as a substrate, conversion of EPA to 12-OH 
product with $\sim 40 \%$ yield can be achieved with TM-FA-HY2, compared to $6 \%$ conversion with pure substrate. Similar pattern was also observed with N. gaditana, although the analysis was complicated with peak overlap of $12-\mathrm{OH}$ product of EPA and $15-\mathrm{OH}$ product of AA. A less pronounced, but a similar increase in conversion was also observed for DHA, especially for $P$. lutheri extract (Figure 5C). Higher conversion levels were also obtained for 10,13-dihydroxyoctadecanoic acid production from LA (Figure 5F). Conversion profiles with other FAs were mostly similar to pure substrate conversion profiles (Figure 5E, Tables S15-S16).

It is important to note that microalgae FA extract contains $25-30 \%$ saturated FAs. One might consider that such a high concentration of saturated FAs could have inhibitory effects through nonproductive binding at the active site. However, no significant inhibition for the production of HFAs, at least in our assay time-scale, has been observed. Our results suggest that microalgae can be used as a convenient source to produce a variety of HFA products and even more effectively than pure substrates in the case of long chain PUFAs. 
A

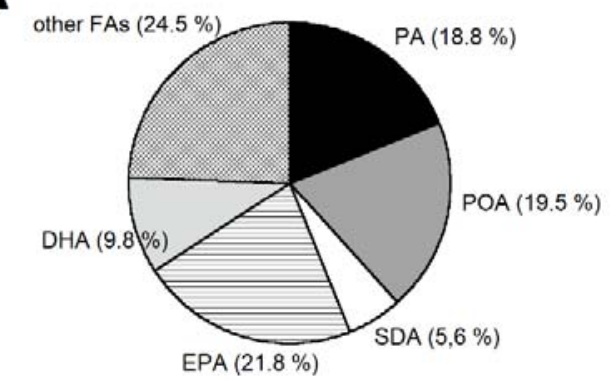

N. gaditana

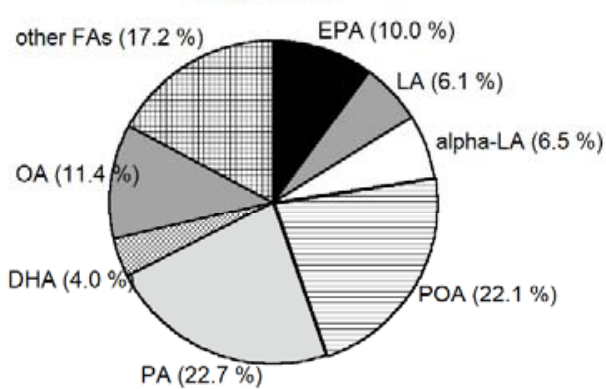

C

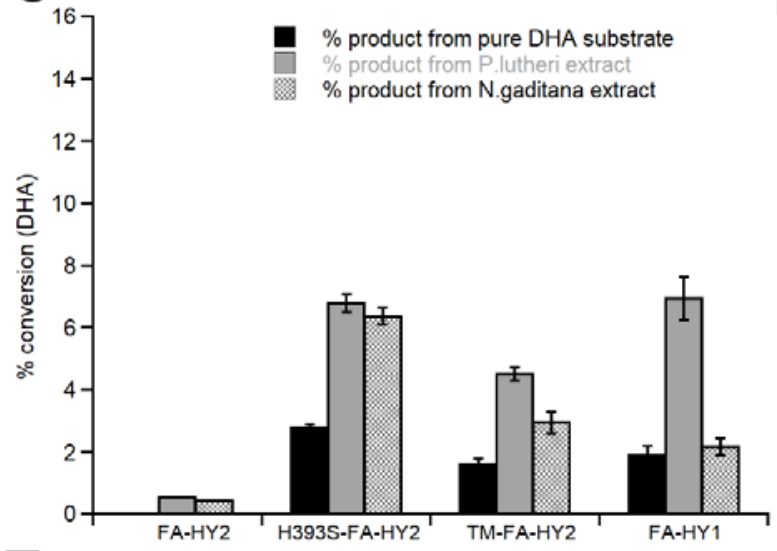

E

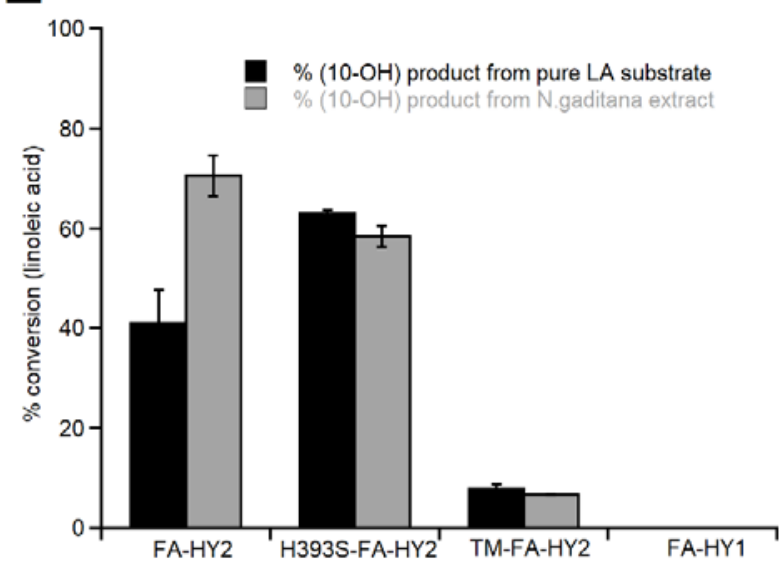

B

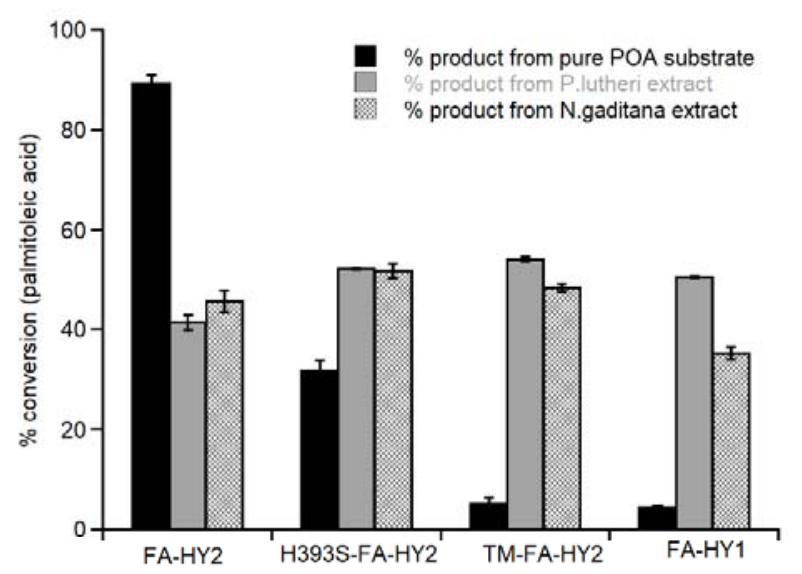

D

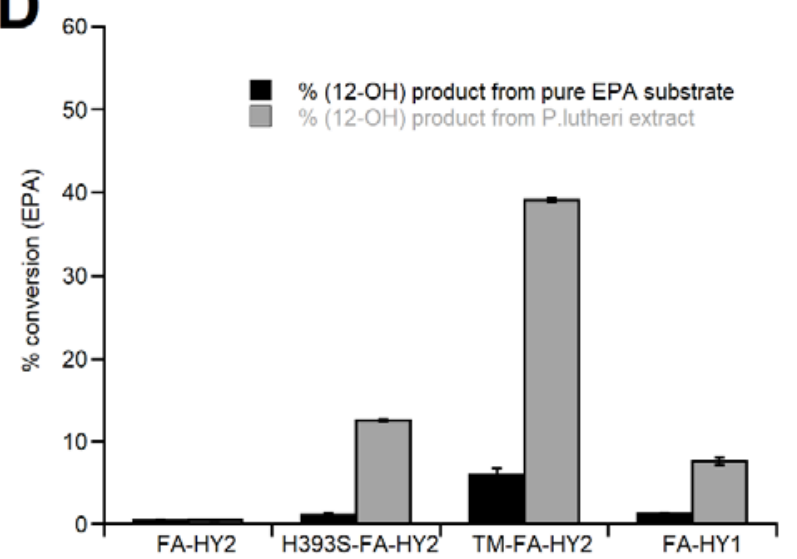

$F$

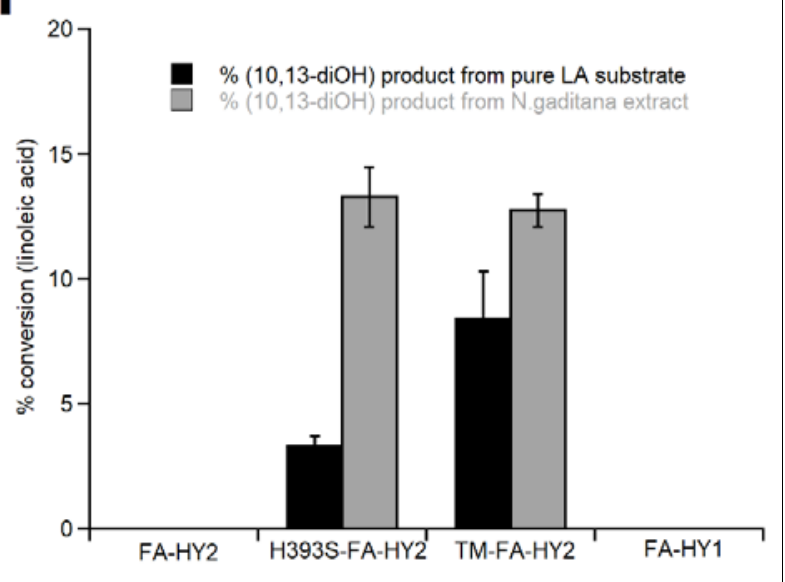


Figure 5. A) FA profiles (weight percentage) of P. lutheri and $N$. gaditana, showing the major FAs present in the FA extract used for assays. PA, palmitic acid; SDA, stearidonic acid. Comparison for the substrate conversion levels of; B) palmitoleic acid; C) DHA; D) EPA; and E and F) LA, when either pure substrate or microalgae extract were used for enzymatic conversion with FA-HY1, FA-HY2, H393S-FA-HY2 and TM-FA-HY2 enzymes. Assays were carried out for 16 hours at $37^{\circ} \mathrm{C}$ by shaking at $110 \mathrm{rpm}$ under anaerobic conditions. Enzyme concentration was $0.3 \mathrm{mg} / \mathrm{mL}$ and substrate concentration was $0.5 \mathrm{mg} / \mathrm{mL}$ for pure substrate and $1 \mathrm{mg} / \mathrm{mL}$ for extracts (total FA concentration), in a total volume of $1 \mathrm{~mL}$. Percentages of each fatty acid in the total extract are given in Supporting Information (Tables S13 and S14).

\section{DISCUSSION}

In this study, we report comparison of activity, kinetics, substrate specificity and regioselectivity of FA-HY1, FA-HY2 and FA-HY2 mutants, where critical active-site residues of FA-HY2 were swapped to the corresponding residues of FA-HY1. Our results clearly demonstrate that three amino acid residues, carboxylate-anchoring residues of T391, H393 and an unexpected nearby residue I378, have remarkable impact for modulating substrate specificity and regioselectivity. Among single residue mutants, changes in activity and regioselectivity were observed with H393SFA-HY2 mutant whereas T391S-FA-HY2 was similar to FA-HY2, which can be attributed to larger size of histidine with respect to threonine. In most cases, combination of mutations yielded a synergistic effect. Especially, DM-FA-HY2 and TM-FA-HY2 exhibited remarkable regioselectivity shift with LA. In addition, both mutants exhibited much higher activity levels with C20 FAs. Besides, no substantial loss in the activity of mutants (except I378P) towards OA, alpha-

and gamma-linolenic acids was observed. Furthermore, kinetic results suggests that although substrate binding is not affected to a great extent, positioning of the substrate with respect to catalytic residues leads to differences in activity levels. 
Preparative scale reactions showed varying levels of conversion and isolated yields (Table S7). We observed that conversions with some of the substrates, especially EPA and ETA, were much higher than the small scale $(1 \mathrm{~mL})$ activity assays. Although this can be partly attributed to increased enzyme concentration and reaction time, other effects such as more efficient mass transfer in large shake flasks vs. small test tubes, should also be responsible. Dispersion of poorly soluble long chain fatty acids in aqueous media are apparently enhanced by quality of mixing leading to efficient accessibility of the substrate to the enzyme. 12-OH product of ETA and 12-OH product of EPA, two new products only accessible by TM-FA-HY2 at a reasonable level, has been converted at $92 \%$ and $69 \%$, respectively. These levels are significantly higher than small scale assays, which exhibited about $6-7 \%$ conversion. $12-\mathrm{OH}$ product of EPA was isolated with $31 \%$ yield and 12-OH product of ETA was isolated with 53\% yield, both with excellent regioselectivity and high stereoselectivity. Wild-type FA-HY2 exhibited $98.5 \%$ conversion and $82 \%$ isolated yield for OA, while wild-type FA-HY1 converted $80 \%$ of LA to $13-\mathrm{OH}$ substrate and an isolated yield of $29 \%$ was achieved. These results show that various HFA products can be prepared from wildtype and mutant enzymes at preparative scale and isolated with good yields in enantiopure form. Since HFA derivatives of C20 FAs are important lipid mediator molecules with anti-inflammatory activity and therapeutic potential ${ }^{[5 a]}$, further engineering of hydratases can contribute to green stereoselective routes for enzymatic synthesis of desired hydroxy derivatives of EPA and AA for medical research. Moreover, all C18 and C20 HFAs produced by wild-type and mutant enzymes can be tested for authentic biological activity alone or as components of FAHFAs ${ }^{[4 c]}$, as well as for their physico-mechanical properties for various bioplastic applications ${ }^{[9 a]}$.

Based on our results, it can be concluded that less bulky carboxylate-anchoring residues (serines vs. threonine and histidine) will possibly pull the FA chain more towards the carboxylate end of 
the channel, with proline 380 helping by pushing the hydrophobic FA chain towards serine residues. This apparently leads to a poorer alignment of the double bonds in closer proximity to the carboxylate (such as $\Delta 9$ and $\Delta 11$ ), but a better alignment of the distant double bonds (such as $\Delta 12$, $\Delta 13$ and $\Delta 14$ ), with respect to catalytic residues (glutamate and tyrosine). In addition, longer activesite cavity with smaller carboxylate-anchoring residues enable acceptance of C20 FAs as efficient substrates. Structure of the substrate also seems to be important besides the size of the channel. This is well demonstrated in the completely swapped activity pattern of FA-HY2 and FA-HY1 towards OA and cis-vaccenic acid (vide supra). Our substrate docking studies with homology models of wild-type enzymes and TM-FA-HY2 support our experimental observations regarding positioning of catalytic residues with respect to double bond.

In cases where multiple products are generated, two scenarios can be considered. First, two different binding modes might be in equilibrium, leading to a distinct product for each binding mode. Other possibility is that there is only one dominant binding mode, but the catalytic residues are sufficiently close to multiple double bond positions to carry out hydration to generate multiple products. Both scenarios are well demonstrated in LA docking models of TM-FA-HY2 (Figure 4B and $4 \mathrm{C})$.

An interesting result from our studies is that I378P mutation almost completely abolished the activity of FA-HY2 towards all substrates. I378 resides at the junction of a beta-sheet and a loop region, which is close to carboxylate-anchoring residues and the substrate. One possibility is that I378P mutation may cause a local conformational change due to sterical restrictions of proline ${ }^{[28]}$, that is detrimental to activity. However, our homology models with TM-FA-HY2 and with FAHY1 shows that mutated proline and its surrounding retains the same position as in wild-type FAHY2. In this model, due to sterical restrictions, proline had to point directly towards FA chain, 
making it closer to the carboxylate end of the substrate channel compared to isoleucine (Figure 4A). This possibly indicates that proline is involved in proper orientation of the carboxylate end of FA substrate in the larger space created by smaller serine residues. Loss of activity upon I378P mutation can very well be rescued in TM-FA-HY2 variant. In fact, a sequence comparison of multiple FAH homologs from HFam2 family (1188 homologs, including FA-HY1 and FA-HY2) of Hydratase Engineering Database ${ }^{[15 c]}$ indicates that homologs with a proline residue instead of an isoleucine mostly accommodate two serines at carboxylate anchoring positions, or at least one serine corresponding to N438 position.

Recently, two studies were published where engineering of the fatty acid hydratase OhyA enabled hydration of carbon-carbon double bonds in non-FA substrates. In the first study, Hauer and coworkers demonstrated hydration of short to medium chain 1-alkenes (C5 to C10) using a combined approach of semi-rational engineering and decoy molecule strategy ${ }^{[11 c]}$. When heptadecanoic acid or similar chain length acids were used as decoy molecules (to activate enzyme), 1-decene was hydrated efficiently by the wild-type enzyme to produce (S)-2-decanol. However, wild-type enzyme had very low or no activity against shorter chain 1-alkenes (C5-C8). Site-saturation mutagenesis of Alanine 248 residue (A248), which points against the omega end of the natural FA substrate, revealed variants that can efficiently hydrate shorter chain 1-alkenes. Mutants with larger residues of leucine or tryptophan (A248L and A248W) were able to efficiently hydrate 1-heptene and 1-hexene, respectively, to produce corresponding secondary alcohols. This has been attributed to pushing effect of the larger residue to shift the alkene substrate more towards the catalytic residues, resulting in better alignment with the nucleophilic water and catalytic residues. Moreover, A248L variant was also able to hydrate functionalized alkenes, internal alkenes, a bulky alkene with an aromatic group and an alkyne. In both FA-HY1 and FA-HY2, 
A248 corresponds to a charged histidine residue (H209 in FA-HY1 and H207 in FA-HY2). In fact, A248 in OhyA seems to have a similar role to I378 in FA-HY2 (or P380 in FA-HY1), by pushing the FA chain to a certain direction within the active site to effect reaction outcome by changing the alignment of double bonds with catalytic residues. In a more recent study, Pichler and coworkers performed enzyme engineering also on OhyA, this time on the residues at the carboxylate end of the substrate ${ }^{[16]}$. Their purpose was to convert key charged residues on the carboxylate end of the FA into residues that will interact favorably with FA head groups other than the free carboxylates, such as esters, amides and alcohols, thus enabling hydration of FA derivatives. Conversion of three residues into alanines (Q265A/T436A/N438A triple mutant) had a large positive impact on the hydration activity towards oleic acid esters compared to wild-type OhyA. T436 and N438 correspond to T391 and H393 in FA-HY2, respectively, and mutations into alanine has possibly opened up more space for bulkier esters as well as created a hydrophobic environment favoring binding of ester head group. Furthermore, oleamide was also more efficiently hydrated with Q265S/N438A double mutant. Both these two recent studies and our study reveal the hot spot residues important for substrate promiscuity. These studies together show that FA hydratases are promiscuous enzymes which can be efficiently directed for hydration reactions of various FAs as well as non-FA substrates to generate non-natural products. An iterative engineering approach of the hot spots at the carboxylate end and at the omega end of FA-HY1, which is already naturally a promiscuous enzyme, should enable access to new substrates and products. While natural FA substrates will be advantageous for feasible production of value-added HFAs from cheap abundant sources of FAs such as microalgae, utilization of non-FA substrates by engineered hydratases will render new selective synthetic approaches for organic synthesis. 
This study is a first demonstration of microalgae oil as a sustainable source of FAs for efficient enzymatic conversion to HFAs. Both wild-type enzymes and mutants can retain most of their properties observed with pure substrates, including substrate specificity and regioselectivity, when a complex mixture of FAs from a microalgae extract is supplied as the substrate. Presence of 25$30 \%$ saturated FAs did not seem to inhibit activity to any significant extent. Moreover, conversion levels of EPA and DHA, two abundant PUFAs in microalgae oil, are even increased for almost all enzymes compared to activity with pure substrates. Given that no significant hydration activity has been reported to date against EPA and DHA with a hydratase, our results render promising opportunities to generate novel HFA derivatives from microalgae as a sustainable source of omega-3 FAs.

\section{CONCLUSION}

Our study provides an important navigation point for generating hydratase variants with desired activity and selectivity for production of a variety of HFAs for use in applications that range from monomer production for bioplastics to synthesis of bioactive compounds with high therapeutic potential. Our TM-FA-HY2 variant is a good representative of this potential with its properties that are unlike FA-HY1 and FA-HY2. TM-FA-HY2 is the only hydratase to date that can convert AA to 12-OH FA product. Unlike other hydratases, TM-FA-HY2 can generate three products from LA, two monohydroxy and one dihydroxy product. Moreover, TM-FA-HY2 exhibited high conversion rates towards EPA and ETA in preparative scale and enabled isolation of novel 12-OH products from EPA and ETA with moderate yields, showing the practical potential of the enzyme for production. Replacement of other amino acid residues at positions targeted in this study by semirational and random enzyme engineering approaches should make it possible to create tailor-made 
enzymes to access specific HFA products. Optimization of reaction conditions will lead to sustainable production of desired HFAs from microalgae as a convenient feedstock.

\section{ASSOCIATED CONTENT}

\section{Supporting Information}

Full scale representative GC chromatogram, Michaelis-Menten plots of all steady-state kinetic data and steady-state kinetic assay conditions, GC-MS spectra of all hydroxy fatty acid products reported in this study, NMR spectra of purified products, absolute configuration analysis, details of preparative scale assays, primer sequences used for site-directed mutagenesis, protein sequences, average conversion percentages of all substrates tested with standard error values, fatty acid composition of microalgae oil, average conversion levels of microalgae fatty acids with standard errors.

\section{AUTHOR INFORMATION}

\section{Corresponding Author}

*Zheng Guo; e-mail: guo@eng.au.dk

\section{Author Contributions}

The manuscript was written through contributions of all authors. All authors have given approval to the final version of the manuscript.

\section{Notes}

The authors declare no competing financial interest. 


\section{ACKNOWLEDGMENT}

This work was supported by Novo Nordisk Foundation (grant NNF16OC0021740) and by AUFF-NOVA from Aarhus Universitets Forskningsfond (AUFF-E-2015-FLS-9-12).

Keywords: Protein Engineering, Enzyme Catalysis, Fatty Acid Hydratase, Hydroxy Fatty Acid, Microalgae

\section{REFERENCES}

[1] a) J. Ogawa, Eur. J. Lipid Sci. Technol. 2015, 117, 577-578; b) P. Bergamo, D. Luongo, J. Miyamoto, E. Cocca, S. Kishino, J. Ogawa, S. Tanabe, M. Rossi, J. Funct. Foods 2014, 11, 192-202.

[2] a) K. R. Kim, D. K. Oh, Biotechnol. Adv. 2013, 31, 1473-1485; b) A. Hiseni, I. W. C. E. Arends, L. G. Otten, ChemCatChem 2015, 7, 29-37; c) A. Beopoulos, J. Verbeke, F. Bordes, M. Guicherd, M. Bressy, A. Marty, J. M. Nicaud, Appl. Microbiol. Biotechnol. 2014, 98, 251-262; d) D. G. Hayes, J. Am. Oil Chem. Soc. 2004, 81, 1077-1103.

[3] J. Miyamoto, T. Mizukure, S. B. Park, S. Kishino, I. Kimura, K. Hirano, P. Bergamo, M. Rossi, T. Suzuki, M. Arita, J. Ogawa, S. Tanabe, J. Biol. Chem. 2015, 290, 2902-2918.

[4] a) M. M. Yore, I. Syed, P. M. Moraes-Vieira, T. Zhang, M. A. Herman, E. A. Homan, R. T. Patel, J. Lee, S. Chen, O. D. Peroni, A. S. Dhaneshwar, A. Hammarstedt, U. Smith, T. E. McGraw, A. Saghatelian, B. B. Kahn, Cell 2014, 159, 318-332; b) O. Kuda, M. Brezinova, M. Rombaldova, B. Slavikova, M. Posta, P. Beier, P. Janovska, J. Veleba, J. Kopecky, E. Kudova, T. Pelikanova, J. Kopecky, Diabetes 2016, 65, 2580-2590; c) B. Kahn, M. Yore, I. Syed, P. Moraes-Vieira, T. Zhang, M. Herman, E. Homan, J. Lee, S. Chen, O. Peroni, A. Hammarstedt, R. Patel, T. McGraw, U. Smith, A. Saghatelian, FASEB J. 2016, 30, 504.503-504.503.

[5] a) M. L. Sulciner, C. N. Serhan, M. M. Gilligan, D. K. Mudge, J. Chang, A. Gartung, K. A. Lehner, D. R. Bielenberg, B. Schmidt, J. Dalli, E. R. Greene, Y. Gus-Brautbar, J. Piwowarski, T. Mammoto, D. Zurakowski, M. Perretti, V. P. Sukhatme, A. Kaipainen, M. W. Kieran, S. Huang, D. Panigrahy, J. Exp. Med. 2018, 215, 115-140; b) M. G. Duvall, B. D. Levy, Eur. J. Pharmacol. 2016, 785, 144-155; c) G. Fredman, M. Spite, Mol. Aspects Med. 2017, 58, 65-71; d) M. Spite, J. Claria, C. N. Serhan, Cell Metab. 2014, 19, 21-36; eC. N. Serhan, Nature 2014, 510, 92-101.

[6] a) B. A. Black, C. Sun, Y. Y. Zhao, M. G. Ganzle, J. M. Curtis, J. Agric. Food Chem. 2013, 61, 5338-5346; b) B. A. Black, E. Zannini, J. M. Curtis, M. G. Ganzle, Appl. Environ. Microbiol. 2013, 79, 1866-1873.

[7] N. d. L. da Silva, M. R. W. Maciel, C. B. Batistella, R. M. Filho, in Twenty-Seventh Symposium on Biotechnology for Fuels and Chemicals (Eds.: J. D. McMillan, W. S. Adney, J. R. Mielenz, K. T. Klasson), Humana Press, Totowa, NJ, 2006, pp. 405-414. 
[8] T. Mi, Y. Dong, U. Santhanam, N. Huang, Exp. Dermatol. 2018, 0.

[9] a) C. Liu, F. Liu, J. Cai, W. Xie, T. E. Long, S. R. Turner, A. Lyons, R. A. Gross, Biomacromolecules 2011, 12, 3291-3298; b) S. Huf, S. Krügener, T. Hirth, S. Rupp, S. Zibek, Eur. J. Lipid Sci. Technol. 2011, 113, 548-561; c) T. Tran, P. Kumar, H.-R. Kim, C. Hou, B. Kim, Polymers 2018, 10, 927.

[10] D. S. Ogunniyi, Bioresour. Technol. 2006, 97, 1086-1091.

[11] a) V. Resch, U. Hanefeld, Catal. Sci. Technol. 2015, 5, 1385-1399; b) C. N. Cornell, M. S. Sigman, Inorg. Chem. 2007, 46, 1903-1909; c) R. M. Demming, S. C. Hammer, B. M. Nestl, S. Gergel, S. Fademrecht, J. Pleiss, B. Hauer, Angew. Chem., Int. Ed. Engl. 2019, 58, 173-177.

[12] M. Engleder, H. Pichler, Appl. Microbiol. Biotechnol. 2018, 102, 5841-5858.

[13] A. J. Boersma, D. Coquiere, D. Geerdink, F. Rosati, B. L. Feringa, G. Roelfes, Nat. Chem. 2010, 2, 991-995.

[14] S.-Q. Wang, Z.-W. Wang, L.-C. Yang, J.-1. Dong, C.-Q. Chi, D.-N. Sui, Y.-Z. Wang, J.-G. Ren, M.-Y. Hung, Y.-Y. Jiang, J. Mol. Catal. A: Chem. 2007, 264, 60-65.

[15] a) L. E. Bevers, M. W. Pinkse, P. D. Verhaert, W. R. Hagen, J. Bacteriol. 2009, 191, 50105012; b) R. M. Demming, M. P. Fischer, J. Schmid, B. Hauer, Curr. Opin. Chem. Biol. 2018, 43, 43-50; c) J. Schmid, L. Steiner, S. Fademrecht, J. Pleiss, K. B. Otte, B. Hauer, J. Mol. Catal. B: Enzym. 2016, 133, S243-S249; d) R. M. Demming, K. B. Otte, B. M. Nestl, B. Hauer, ChemCatChem 2017, 9, 758-766; e) K. Zorn, I. Oroz-Guinea, H. Brundiek, U. T. Bornscheuer, Prog. Lipid Res. 2016, 63, 153-164.

[16] M. Engleder, G. A. Strohmeier, H. Weber, G. Steinkellner, E. Leitner, M. Muller, D. Mink, M. Schurmann, K. Gruber, H. Pichler, Angew. Chem., Int. Ed. Engl. 2019.

[17] M. Engleder, T. Pavkov-Keller, A. Emmerstorfer, A. Hromic, S. Schrempf, G. Steinkellner, T. Wriessnegger, E. Leitner, G. A. Strohmeier, I. Kaluzna, D. Mink, M. Schurmann, S. Wallner, P. Macheroux, K. Gruber, H. Pichler, ChemBioChem 2015, 16, $1730-1734$.

[18] a) A. Hirata, S. Kishino, S. B. Park, M. Takeuchi, N. Kitamura, J. Ogawa, J. Lipid Res. 2015, 56, 1340-1350; b) K. R. Kim, H. J. Oh, C. S. Park, S. H. Hong, J. Y. Park, D. K. Oh, Biotechnol. Bioeng. 2015, 112, 2206-2213.

[19] C. S. Chaurasia, T. D. Williams, C. M. Judson, R. P. Hanzlik, J. Mass Spectrom. 1995, 30, 1018-1022.

[20] T. R. Hoye, C. S. Jeffrey, F. Shao, Nat. Protoc. 2007, 2, 2451-2458.

[21] R. R. Guillard, in Culture of Marine Invertebrate Animals (Eds.: W. L. Smith, M. H. Chanley), Springer, Boston, MA, 1975, pp. 29-60.

[22] E. F. Pettersen, T. D. Goddard, C. C. Huang, G. S. Couch, D. M. Greenblatt, E. C. Meng, T. E. Ferrin, J. Comput. Chem. 2004, 25, 1605-1612.

[23] B. Webb, A. Sali, Curr. Protoc. Bioinformatics 2014, 47, 5 6 1-32.

[24] S. C. Lovell, I. W. Davis, W. B. Arendall, 3rd, P. I. de Bakker, J. M. Word, M. G. Prisant, J. S. Richardson, D. C. Richardson, Proteins 2003, 50, 437-450.

[25] O. Trott, A. J. Olson, J. Comput. Chem. 2010, 31, 455-461.

[26] a) P. W. Behrens, D. J. Kyle, J. Food Lipids 1996, 3, 259-272; b) D. Gangl, J. A. Zedler, P. D. Rajakumar, E. M. Martinez, A. Riseley, A. Wlodarczyk, S. Purton, Y. Sakuragi, C. J. Howe, P. E. Jensen, C. Robinson, J. Exp. Bot. 2015, 66, 6975-6990.

[27] a) J. Camacho-Rodriguez, M. C. Ceron-Garcia, J. M. Fernandez-Sevilla, E. Molina-Grima, Algal Res. 2015, 11, 63-73; b) J. P. Schwarzhans, D. Cholewa, P. Grimm, U. Beshay, J. M. 
Risse, K. Friehs, E. Flaschel, J. Appl. Phycol. 2015, 27, 1389-1399; c) H. Safafar, M. Z. Hass, P. Moller, S. L. Holdt, C. Jacobsen, Mar. Drugs 2016, 14.

[28] M. W. MacArthur, J. M. Thornton, J. Mol. Biol. 1991, 218, 397-412.

Table of Contents Entry (for Layout $1-5,5 \mathrm{~cm}$ x $5,0 \mathrm{~cm}$ )

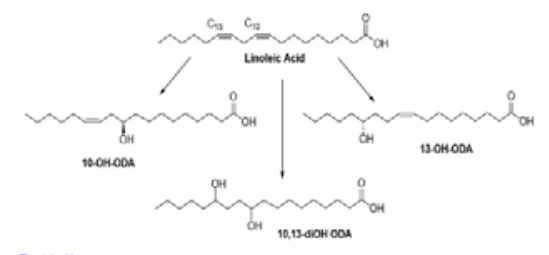

;

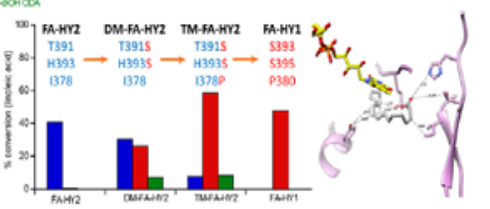

Text for Table of contents:

Promiscuity dictated by a few residues: Comparative analysis of the active site residues of two Fatty Acid Hydratases from L. Acidophilus, which have high sequence homology but distinct substrate specificity and regioselectivity, reveal three critical amino acids that play remarkable role in directing substrate specificity and regioselectivity of hydration. 